\title{
FORMATION OF WATER IN THE WARM ATMOSPHERES OF PROTOPLANETARY DISKS
}

\author{
A. E. Glassgold ${ }^{1}$, R. Meijerink ${ }^{1,2}$, AND J. R. NAJita ${ }^{3}$ \\ ${ }^{1}$ Astronomy Department, University of California, Berkeley, CA 94720, USA; aglassgold@ astro.berkeley.edu \\ ${ }^{2}$ California Institute of Technology, Division of Geological and Planetary Sciences, MS 150-21, Pasadena, CA 91125, USA; rowin@gps.caltech.edu \\ ${ }^{3}$ National Optical Astronomy Observatory, 950 North Cherry Avenue, Tucson, AZ 85719, USA; najita@ noao.edu \\ Received 2009 February 10; accepted 2009 May 26; published 2009 July 21
}

\begin{abstract}
The gas-phase chemistry of water in protoplanetary disks is analyzed with a model based on X-ray heating and ionization of the disk atmosphere. Several uncertain processes appear to play critical roles in generating the column densities of warm water that are detected from disks at infrared wavelengths. The dominant factors are the reactions that form molecular hydrogen, including formation on warm grains, and the ionization and heating of the atmosphere. All of these can work together to produce a region of high water abundances in the molecular transition layer of the inner disk atmosphere, where atoms are transformed into molecules, the temperature drops from thousands to hundreds of Kelvins, and the ionization begins to be dominated by the heavy elements. Grain formation of molecular hydrogen and mechanical heating of the atmosphere can play important roles in this region and directly affect the amount of warm water in protoplanetary disk atmospheres. Thus, it may be possible to account for the existing measurements of water emission from $\mathrm{T}$ Tauri disks without invoking transport of water from cooler to warmer regions. The hydroxyl radical $\mathrm{OH}$ is underabundant in this model of disk atmospheres and requires consideration of additional production and excitation processes.
\end{abstract}

Key words: astrochemistry - planetary systems: protoplanetary disks - X-rays: stars

Online-only material: color figures

\section{INTRODUCTION}

The water molecule plays a significant role in the evolution of protoplanetary disks and in the formation of small and large bodies including planets (e.g., Ciesla \& Cuzzi 2006; Jewitt et al. 2006). Recent results from the Spitzer Space Telescope reveal rich mid-infrared (MIR) water emission spectra from 10-35 $\mu \mathrm{m}$ that appear to be a common characteristic of T Tauri disks (Carr \& Najita 2008; Salyk et al. 2008). Assuming thermal equilibrium populations, typical temperatures and column densities have been estimated to be $T \sim 500-1000 \mathrm{~K}$ and $N\left(\mathrm{H}_{2} \mathrm{O}\right) \sim 10^{17}-10^{18} \mathrm{~cm}^{-2}$, with emitting areas of the order of a few $\mathrm{AU}$ in radius. MIR emission from other molecules ( $\mathrm{HCN}$, $\mathrm{C}_{2} \mathrm{H}_{2}, \mathrm{OH}, \mathrm{CO}_{2}$ ) is also detected with similar temperatures and likely similar emitting radii, e.g., Carr \& Najita (2008). These MIR lines appear to probe lower gas temperatures and larger disk radii than the previously known suite of gaseous inner disk diagnostics. UV fluorescent emission from $\mathrm{H}_{2}$ and near-infrared (NIR) transitions of CO probe warm gas temperatures (1000$3000 \mathrm{~K}$ ) and primarily the inner $<1$ AU of the disk (Najita et al. 2007). Measurements of molecular line emission have been obtained for many other protoplanetary disks by Spitzer and should become available for analysis and interpretation in the near future.

Our earlier model of the thermal-chemical structure of an $\mathrm{X}$-ray irradiated generic $\mathrm{T}$ Tauri disk atmosphere (Glassgold et al. 2004; henceforth GNI04) included the synthesis of $\mathrm{H}_{2} \mathrm{O}$ by temperature-sensitive neutral radical reactions (Table 1 of that paper), but it did not predict strong water emission. Possible explanations for this deficiency might be that water is dredged up from deeper parts of the disk atmosphere by turbulent mixing, or that it is transported radially by inward migration of intermediate-sized bodies and desorbed (Ciesla \& Cuzzi 2006; Najita et al. 2007; Salyk et al. 2008). In this case, measurements of water abundances in disk atmospheres might be used to trace dynamical processes such as turbulence and migration. However, disk gas-phase chemistry is affected by several often poorly known physical processes, so it is important to fully explore nondynamical explanations. In this paper, we analyze some of these processes in light of the recent Spitzer $\mathrm{H}_{2} \mathrm{O}$ observations by exploring alternative or modified chemical pathways that may enhance the abundance of water and other molecules in disk atmospheres. A longer term goal is to attempt to use observations of water to infer the physical properties of protoplanetary disks.

This study complements recent work on disk chemistry by focusing on the thermal-chemical properties of an X-rayirradiated disk atmosphere that has experienced grain growth and settling. X-ray induced chemistry has a long history going back 25 years (Krolik \& Kallman 1983). References to early work is given in the pioneering papers by Lepp \& Dalgarno (1996) and Maloney et al. (1996). The latter calculate the water abundance in X-ray-transition regions, as do Meijerink \& Spaans (2005). Stauber et al. (2005) develop X-ray chemistry for spherically symmetric envelopes around young stellar objects (YSOs) with specific emphasis on water (Stauber et al. 2006).

Many disk chemical models consider the outer region of disks, whereas we concentrate on the inner $<10-20$ AU. Several recent papers treat inner disk gas-phase chemistry (Markwick et al. 2002; Nomura \& Millar 2005; Nomura et al. 2007, 2009; Agúndez et al. 2008; Gorti \& Hollenbach 2008; Woods \& Willacy 2009). Nomura et al. (2007) focus on $\mathrm{H}_{2}$, and Nomura et al. (2009) follow molecules along prescribed stream lines and find that the abundances of many organic molecules change as they enter the inner disk region, notably methanol but not water. Markwick et al. and Agúndez et al. include X-ray ionization in terms of a phenomenological ionization rate, whereas Woods and Willacy use a more complete theory based on Gorti \& Hollenbach (2004). Agúndez et al. carry out time-dependent abundance calculations, in some cases at fixed temperatures. We 
shall discuss the water abundances obtained by these authors in Section 3.

In this paper, we analyze a number of physical processes that can affect the abundance of water in the warm part of the disk atmosphere ( $T>200-300 \mathrm{~K})$ using the GNI04 model. In Section 2, we discuss the role played by dust through the thermal coupling of the gas and dust; the efficiency of the gas-phase reactions that produce $\mathrm{H}_{2}$ and $\mathrm{H}_{2} \mathrm{O}$; the role of $\mathrm{H}_{2}$ and $\mathrm{H}_{2} \mathrm{O}$ formation on dust grains; and finally the effects of enhanced mechanical heating of the atmosphere. Implementation of these processes with our thermal-chemical model, along with new information about their efficiency, indicates that column densities of warm water in the range observed can be obtained without invoking radial or vertical transport. The implications of these results are taken up in Section 3 and in Section 4.

\section{THERMAL-CHEMICAL MODEL OF X-RAY-IRRADIATED DISKS}

\subsection{The Basic Model}

This work builds on our earlier model of the thermalchemical structure of the inner disk of a T Tauri star (GNI04; see Meijerink et al. 2008 for corrections and additional applications). The disk is irradiated by stellar $\mathrm{X}$-rays that ionize and heat the disk surface. The X-ray luminosity is $L_{X}=2 \times 10^{30} \mathrm{erg} \mathrm{s}^{-1}$; a thermal spectrum is used with $T_{\mathrm{X}}=1.0 \mathrm{keV}$ and a low-energy cutoff of $E_{0}=200 \mathrm{eV}$. As in GNI04 and our earlier work, cosmic rays are ignored on the basis of their being blown away from the inner disk by the stellar wind. The total gas density distribution and the dust temperature are taken from D'Alessio et al. $(1999,2001)$, and the gas-phase temperature and abundances are solved for self-consistently keeping the density and dust temperature fixed. In a more general model that treats the dust and gas separately, a new equilibrium would be calculated, including the effects of changing the gas temperature on the hydrostatic balance and on the dust temperature.

The gas temperature in the current model is determined by balancing heating from X-rays and mechanical processes with cooling by line radiation. GNI04 employed a simple chemistry with 25 species and 125 reactions that included the heavy atoms, $\mathrm{C}$ and $\mathrm{O}$, as well as $\mathrm{H}$ and He. In order to provide a more complete theory of X-ray ionization, the chemical code used here treats all of the most abundant heavy atoms and includes nearly 400 reactions (R. Meijerink and A. E. Glassgold 2009, in preparation). As mentioned in Section 1, chemical synthesis is achieved by neutral radical reactions with moderate barriers that can be overcome in the warm upper atmosphere of the disk. Ionic reactions also contribute, but their most important role is to destroy molecules. One difference with GNI04 is that we use depleted abundances, defined relative to hydrogen (nuclei) as usual, with $x_{\mathrm{O}}=3.5 \times 10^{-4}$ and $x_{\mathrm{C}}=1.4 \times 10^{-4}$. The thermal part of the program is unchanged from GNI04 even as changes are made in the chemistry. For example, Ne II and water cooling are still ignored, although their inclusion could change the quantitative aspects of the present results. In addition, a significant fraction of the stellar NIR flux may be captured by water molecules. A consistent theory of the thermal effects of water in disk atmospheres requires consideration of both heating and cooling. We postpone treating this important topic to a future study. Also omitted in this pure X-ray-irradiated disk model are the effects of UV radiation, which can affect the abundances of $\mathrm{OH}, \mathrm{H}_{2} \mathrm{O}$, and other species. Efforts are under way to include both UV radiation and X-rays in our models.
Dust grains play a direct role in the thermal balance via the familiar collisional coupling of the dust and the gas. Cooling of the gas by the dust becomes important in the region we refer to as the transition region where the temperature and ionization level are rapidly decreasing and atomic species are transformed into molecules. Since the chemistry is sensitive to temperature, the dust indirectly affects the chemical abundances. In the GNI04 model, it was assumed that the dust in the inner disk had experienced growth and settling. The grain area per $\mathrm{H}$ nucleus is given by the average of $\pi a^{2}$ over the MRN statistical distribution of the grain size $a$ (Equation (A4) of GNI04),

$$
\frac{n_{\mathrm{d}}}{n_{\mathrm{H}}}\left\langle\pi a^{2}\right\rangle=\frac{\rho_{\mathrm{d}} / \rho_{\mathrm{g}}}{a_{\text {geom }}} \frac{m}{(4 / 3) \tilde{\rho}}, \quad a_{\text {geom }}=\left(a_{1} a_{2}\right)^{1 / 2},
$$

where $n_{\mathrm{d}}$ and $\rho_{\mathrm{d}}$ are the volumetric number and mass density of the dust grains, $\rho_{\mathrm{g}}$ is the mass density of gas, $\tilde{\rho}$ is the internal density of a typical grain, $m$ is the mean mass of the gas per $\mathrm{H}$ nucleus, and $a_{1}$ and $a_{2}$ are the minimum and maximum grain radii for an MRN distribution. The key parameter in Equation (1) is the dust-to-gas ratio $\rho_{\mathrm{d}} / \rho_{\mathrm{g}}$ divided by $a_{\text {geom }}$, the geometric mean of $a_{1}$ and $a_{2}$. In GNI04, these parameters were set at $\rho_{\mathrm{d}} / \rho_{\mathrm{g}}=0.01$ and $a_{\text {geom }}=0.707 \mu \mathrm{m}$, following Wood et al. (2001). This choice corresponds to a factor of 20 increase in $a_{\text {geom }}$ and a factor of 20 decrease in effective grain surface area relative to the interstellar medium. Wood et al. actually used an MRN distribution with $a_{1}=0.01 \mu \mathrm{m}$, an exponential cutoff at $a=50 \mu \mathrm{m}$ and $a_{2}=1 \mathrm{~mm}$. Had we used the grain model in D'Alessio et al. (2001; $a_{1}=0.005 \mu \mathrm{m}$ and $\left.a_{2}=1 \mathrm{~mm}\right)$, then $a_{\text {geom }}$ would be $2.24 \mu \mathrm{m}$, or 3.2 times larger than in GNI04. In this paper, we follow GNI04 but also consider larger values of $a_{\text {geom }}$, i.e., $a_{\text {geom }}=7.07 \mu \mathrm{m}$ as well as $a_{\text {geom }}=0.707 \mu \mathrm{m}$. This range gives grain areas per $\mathrm{H}$ nucleus that are roughly consistent with the dust depletion deduced by Furlan et al. (2006) for the upper atmospheres of T Tauri disks observed with Spitzer.

\subsection{The Dependence of the Water Abundance on the Physical Properties}

Only gas-phase reactions were considered by GNI04 for the formation of $\mathrm{H}_{2}$ because the grains in disk atmospheres were considered too warm for the usual grain synthesis familiar from the interstellar medium (e.g., Hollenbach \& Salpeter 1971) to be efficient. Instead, $\mathrm{H}_{2}$ was formed by radiative attachment of electrons

$$
\mathrm{e}+\mathrm{H} \rightarrow \mathrm{H}^{-}+h v \quad \mathrm{H}^{-}+\mathrm{H} \rightarrow \mathrm{H}_{2}+\mathrm{e},
$$

and by three-body reactions,

$$
3 \mathrm{H} \rightarrow \mathrm{H}_{2}+\mathrm{H} \quad 2 \mathrm{H}+\mathrm{H}_{2} \rightarrow 2 \mathrm{H}_{2},
$$

the latter accompanied by collisional dissociation,

$$
\mathrm{H}+\mathrm{H}_{2} \rightarrow 3 \mathrm{H} \quad \mathrm{H}_{2}+\mathrm{H}_{2} \rightarrow \mathrm{H}_{2}+2 \mathrm{H} .
$$

These gas-phase reactions led to $\mathrm{H}_{2}$ abundances approaching $\sim 10^{-3}$ in the surface region, a level sufficient to produce significant amounts of warm CO $\left(\sim 10^{17}-10^{18} \mathrm{~cm}^{-2}\right)$ by a sequence of neutral radical reactions that begins with the slightly endothermic reaction

$$
\mathrm{O}+\mathrm{H}_{2} \rightarrow \mathrm{H}+\mathrm{OH}
$$

and leads to water by the exothermic reaction,

$$
\mathrm{OH}+\mathrm{H}_{2} \rightarrow \mathrm{H}_{2} \mathrm{O}+\mathrm{H} \text {. }
$$


Both of these reactions have barriers that require warm conditions to be effective, i.e., $T \gtrsim 200-300 \mathrm{~K}$. OH also leads to $\mathrm{O}_{2}$ and $\mathrm{CO}$ by fast exothermic radical reactions without significant barriers,

$$
\mathrm{OH}+\mathrm{O} \rightarrow \mathrm{O}_{2}+\mathrm{H} \quad \mathrm{C}+\mathrm{OH} \rightarrow \mathrm{CO}+\mathrm{H} .
$$

$\mathrm{OH}$ and $\mathrm{H}_{2} \mathrm{O}$ are mainly destroyed by charge transfer with $\mathrm{H}^{+}$,

$$
\begin{gathered}
\mathrm{H}^{+}+\mathrm{OH} \rightarrow \mathrm{OH}^{+}+\mathrm{H}, \\
\mathrm{H}^{+}+\mathrm{H}_{2} \mathrm{O} \rightarrow \mathrm{H}_{2} \mathrm{O}^{+}+\mathrm{H} .
\end{gathered}
$$

The chemistry defined by the above equations starting with Equation (5) can be approximated by the following formula for the abundance of water,

$$
\frac{x\left(\mathrm{H}_{2} \mathrm{O}\right)}{x(\mathrm{O})} \sim \kappa(T)\left(\frac{x\left(\mathrm{H}_{2}\right)}{x\left(\mathrm{H}^{+}\right)}\right)^{2}
$$

where

$$
\kappa \equiv \frac{k_{5} k_{6}}{k_{8} k_{9}} \approx 10^{-18} T^{4.27} \exp (-4823 / T) .
$$

The ratio of water to atomic oxygen depends quadratically on the ratio of $\mathrm{H}_{2}$ to $\mathrm{H}^{+}$because the synthesis of water proceeds via two radical reactions with $\mathrm{H}_{2}$, (Equations (5) and (6)), and because the precursor radical $\mathrm{OH}$ as well as $\mathrm{H}_{2} \mathrm{O}$ are destroyed primarily by fast charge exchange with $\mathrm{H}^{+}$(Equations (8) and (9)). The factor $\kappa(T)$ is sensitive to temperature because the product of the rate coefficients, $k_{5}$ and $k_{6}$, for the two synthesis reactions is temperature sensitive, whereas the product of the two rate coefficients, $k_{8}$ and $k_{9}$, for the two ionic destruction reactions is not. Equation (10) ignores several factors that can limit its accuracy: the role of the backward reactions to Equations (5) and (6) at high temperatures; the contribution of ion-molecule reactions to the synthesis of water; and the destruction of $\mathrm{OH}$ and $\mathrm{H}_{2} \mathrm{O}$ by $\mathrm{He}^{+}$ions.

The main purpose of introducing Equation (10) is to elucidate the underlying physics of the molecular transition and not to reproduce the full model calculations. The main conclusion from this approximate treatment is that the water abundance in disk atmospheres is sensitive to two quantities that vary rapidly in the transition zone between the very warm upper atmosphere and the cool near-midplane region, the temperature and the ratio of the molecular hydrogen abundance to the $\mathrm{H}^{+}$abundance. Any process that affects these quantities can change the abundance of water. For our model of an X-ray-irradiated disk, the X-rays play a key role since they heat and ionize the gas.

The GNI04 model calculations yielded small column densities of warm water $\left(\lesssim 10^{13} \mathrm{~cm}^{-2}\right.$ at $\left.T>200-300 \mathrm{~K}\right)$. In contrast, observations indicate the presence of abundant warm water, e.g., Carr \& Najita (2008) reported $N\left(\mathrm{H}_{2} \mathrm{O}\right)=$ $6.5 \times 10^{17} \mathrm{~cm}^{-2}$ at $T=500-600 \mathrm{~K}$ in AA Tau, and Salyk et al. (2008) found similar columns in AS 205A and DR Tau. According to our calculations, the water is probably located in the vertical thermal-chemical transition region that occurs between the hot and cool parts of the disk, where the gas temperature is relatively high, e.g., in the $300-2000 \mathrm{~K}$ range. The observed water lines are in emission, which further suggests that the lines form above the MIR dust photosphere. The deficiency of the GNI04 model in this respect suggests three possibilities: (1) other processes heat the atmosphere over larger column densities, so that the water deeper down in the atmosphere is put into emission; (2) water is transported dynamically and mixed into the upper atmosphere where it is heated; (3) more efficient chemical pathways form water in the disk atmosphere. We mainly focus on options (1) and (3) in this paper.

\subsection{Gas-phase Reactions}

Reaction (5) is an important destruction mechanism for $\mathrm{H}_{2}$. The recent critical review by Baulch et al. (2005) contains laboratory measurements of this reaction from $300-3500 \mathrm{~K}$. The low-temperature data $(T \gtrsim 300 \mathrm{~K}$ ) are 20 years old and were obtained with shock tubes or flash photolysis. GNI04 used the value recommended in the earlier Baulch et al. (1992) review,

$$
k_{5}=8.5 \times 10^{-20} T^{2.67} e^{-3163 / T} \mathrm{~cm}^{3} \mathrm{~s}^{-1} .
$$

The more recent experiments refer to the higher-temperature regime, $T=1500-3500 \mathrm{~K}$, and the above fit is still satisfactory at lower temperatures. Nonetheless, considerable uncertainty must be attached to Equation (5) ( 50\%-100\%), and even more when it is extrapolated below $300 \mathrm{~K}$. GNI04 mistakenly used the exponent 1.67 instead of 2.67, seriously underestimating the production of oxygen molecules while underestimating the destruction of $\mathrm{H}_{2}$ via Equation (5). This error was corrected in MGN08, but even with this change only a small amount of warm water was generated, e.g., $N\left(\mathrm{H}_{2} \mathrm{O}\right) \lesssim 10^{13} \mathrm{~cm}^{-2}$ at $1 \mathrm{AU}$. Significant water abundances, say $x\left(\mathrm{H}_{2} \mathrm{O}\right)>10^{-6}$, were only achieved at large vertical column densities $\left(N_{\mathrm{H}}>10^{22} \mathrm{~cm}^{-2}\right)$ where the dust temperature is barely above freeze-out. This situation is illustrated by the dotted curves in the middle and bottom panels of Figure 1 for a radial distance of $1 \mathrm{AU}$.

Another critical gas-phase process is the three-body formation of $\mathrm{H}_{2}$, the reactions in Equation (3). In the past, these threebody rate coefficients were obtained with detailed balance from measurements of the inverse reactions, usually those of Jacobs et al. (1967). Flower \& Harris (2007) have used new equilibrium constants to obtain three-body formation rate coefficients that are significantly larger than those given in the review of Cohen \& Westberg (1983) and used by GNI04. For example, the first forward reaction in Equation (3) is, as given by Flower and Harris,

$$
k_{3}\left(\mathrm{H} \rightarrow \mathrm{H}_{2}+\mathrm{H}\right)=1.44 \times 10^{-26} T^{-1.54} \mathrm{~cm}^{6} \mathrm{~s}^{-1},
$$

which is to be compared with the Cohen and Westberg rate, $8 \times 10^{-33} \mathrm{~cm}^{6} \mathrm{~s}^{-1}$.

The top panel of Figure 1 shows the change in the abundances of hydrogenic species obtained using the new rate coefficients. The difference between old and new rate coefficients is not manifest until $N_{\mathrm{H}}>5 \times 10^{20} \mathrm{~cm}^{-2}$ because the $\mathrm{H}_{2}$ formation proceeds mainly via the $\mathrm{H}^{-}$route, Equation (2), at high altitudes. The strong rise in the $\mathrm{H}_{2}$ abundance as $N_{\mathrm{H}}$ approaches $5 \times 10^{20} \mathrm{~cm}^{-2}$ occurs because the three-body rate increases rapidly with decreasing temperature in a region where the atomic $\mathrm{H}$ density is also increasing toward $10^{10} \mathrm{~cm}^{-3}$. As a result, the $\mathrm{H}-\mathrm{H}_{2}$ transition is shifted to smaller vertical column densities, which means to warmer regions higher in the atmosphere. As can be seen Figure 1, this also leads to a significant increase in the amount of warm water, aided by a drop in the abundance of $\mathrm{H}^{+}$ according to Equations (8) and (9). The incomplete transitions of atomic $\mathrm{O}$ to $\mathrm{OH}$ and $\mathrm{H}_{2} \mathrm{O}$ (to abundances $\sim 10^{-6}$ ) now occur in the same general region as the hydrogen and carbon transitions. The solid curves in Figure 1 define the first in a series of comparison models to be introduced in this section ("model 1"). The other models are defined in Table 1 and their 


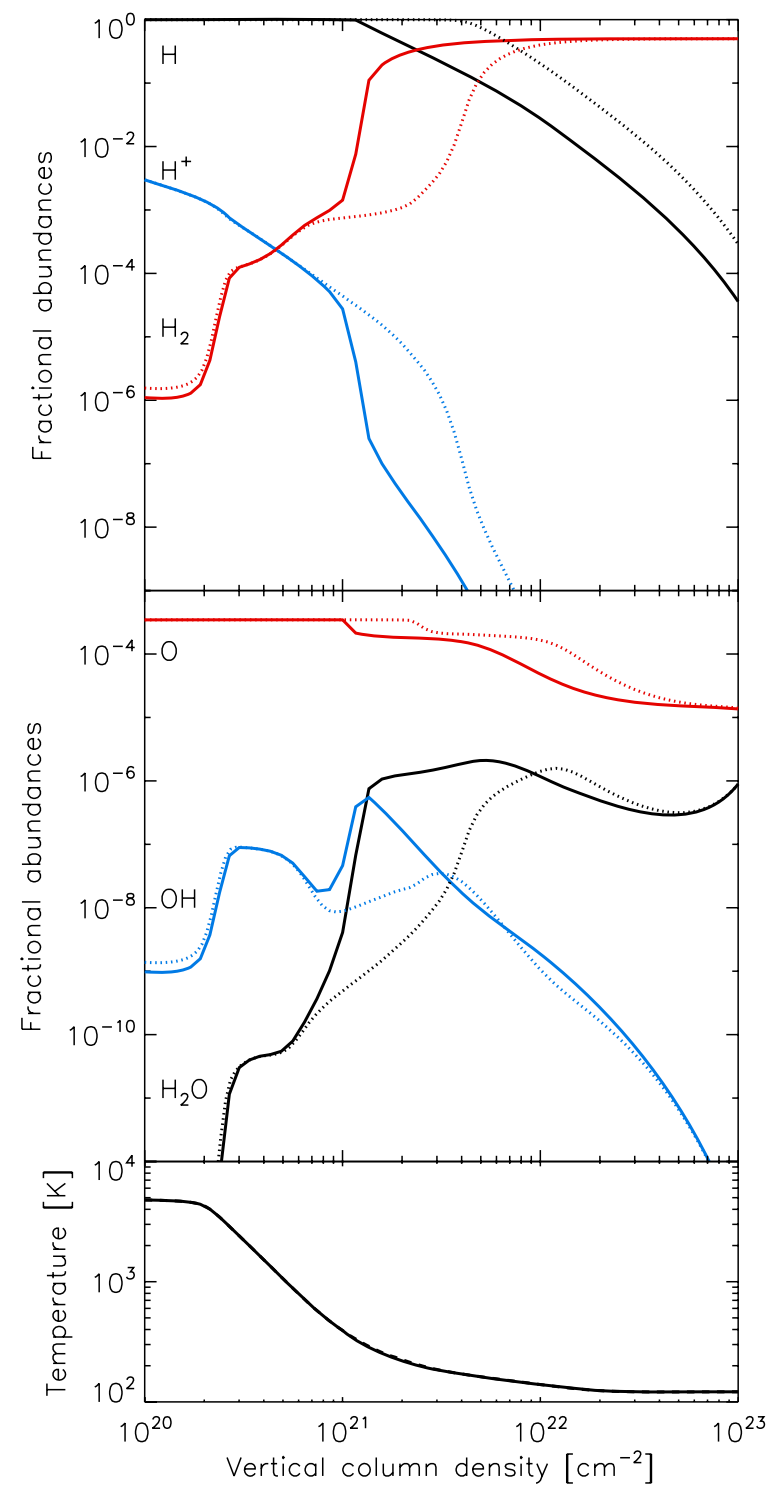

Figure 1. Hydrogen (top) and oxygen (middle) species at 1 AU plotted vs. vertical column density for old (dotted line) and new (solid line) three-body reaction rate coefficients discussed in GNI04 (dotted line) and this paper (solid line). See Section 2.3 for details. With the new reaction rates, the $\mathrm{H}-$ $\mathrm{H}_{2}$ transition, where $x(\mathrm{H})=x\left(\mathrm{H}_{2}\right)$, is shifted upward by a factor of 3 in vertical column from $N_{\mathrm{H}}=7 \times 10^{21} \mathrm{~cm}^{-2}$ to $N_{\mathrm{H}}=2 \times 10^{21} \mathrm{~cm}^{-2}$. The transition to water is also similarly shifted upward. The solid curves represent model 1 in Tables 1 and 2 .

(A color version of this figure is available in the online journal.)

specific properties are given later in Table 2. Note that the parameter $a_{\text {geom }}$ listed in Table 1 determines the dust cooling of the gas as well as the formation of $\mathrm{H}_{2}$ on grains.

\subsection{The Formation of $\mathrm{H}_{2}$ on Dust Grains}

As discussed in Section 2.2, we previously considered only gas-phase pathways for forming $\mathrm{H}_{2}$ on the assumption that dust grains in disk atmospheres are too warm for efficient grain synthesis. This idea is well founded in the standard theory of $\mathrm{H}_{2}$ formation in interstellar clouds (e.g., Hollenbach \& Salpeter 1971) and substantiated by low-temperature laboratory experiments using interstellar dust analogs and interpreted with theoretical models based on the physical adsorption of H atoms (e.g., Vidali et al. 2004; Perets et al. 2005, 2007).
Table 1

Chemical Models

\begin{tabular}{llcc}
\hline \hline Model & \multicolumn{1}{c}{ Chemistry } & $a_{\text {geom }}(\mu \mathrm{m})$ & $\alpha_{\mathrm{h}}$ \\
\hline 1 & Pure gas phase & 0.707 & 0.01 \\
2 & $\mathrm{H}_{2}$ Formation on grains & 0.707 & 0.01 \\
3 & $\mathrm{H}_{2}$ Formation on grains & 7.070 & 0.01 \\
4 & $\mathrm{H}_{2} \& \mathrm{H}_{2}$ O Formation on grains & 0.707 & 0.01 \\
5 & Pure gas phase & 0.707 & 1.00 \\
6 & $\mathrm{H}_{2}$ Formation on grains & 0.707 & 1.00 \\
\hline
\end{tabular}

The efficiency of $\mathrm{H}_{2}$ formation is close to unity in a narrow range (width $\sim 5 \mathrm{~K}$ ) of temperature near $15 \mathrm{~K}$, depending on the experimental sample. At lower temperatures, incident $\mathrm{H}$ atoms are insufficiently mobile, and at higher temperatures they leave the surface before forming molecules. Invoking a model based on chemical adsorption, Cazaux and Tielens found that $\mathrm{H}_{2}$ can form with moderate efficiency $(\lesssim 0.2)$ on warm dust with temperatures up to $900 \mathrm{~K}$ (Cazaux \& Tielens 2002, 2004; Cazaux et al. 2005). We adopt this model here because it is a reasonable extension of existing understanding and because it is supported by laboratory experiments with graphite at an intermediate temperature of a few hundred Kelvins (e.g., Zecho et al. 2002; Hornekær et al. 2006a, 2006b).

We add grain formation to the program described earlier by writing the $\mathrm{H}_{2}$ formation rate per unit volume in the standard form, i.e., 1/2 times the destruction rate of atomic $\mathrm{H}$ :

$$
R=\frac{1}{2} n(\mathrm{H}) v(\mathrm{H}) n_{\mathrm{d}}\left\langle\pi a^{2}\right\rangle \in S\left(T_{\mathrm{g}}, T_{\mathrm{d}}\right) .
$$

The two factors after the $1 / 2$ give the incident $\mathrm{H}$ atom flux, the next two specify the grain surface area per unit volume, and the last two are the product of the formation efficiency and the sticking probability (or, more simply, the overall efficiency). We use GNI04 for the mean grain area per unit volume, based on the MRN distribution and an internal dust grain density of $3 \mathrm{~g} \mathrm{~cm}^{-3}$, to obtain (in units of $\mathrm{cm}^{3} \mathrm{~s}^{-1}$ ),

$$
R=4.32 \times 10^{-19} T_{\mathrm{g}}^{1 / 2} n(\mathrm{H}) n_{\mathrm{H}} \epsilon S \times\left(\frac{\rho_{\mathrm{d}} / \rho_{\mathrm{g}}}{0.01}\right)\left(\frac{\mu \mathrm{m}}{a_{g}}\right) .
$$

We use the right panel of Figure 2 of Cazaux et al. (2005) for the formation efficiency $\epsilon$ as a function of the dust temperature $T_{d}$ for the one case they considered where the gas and dust temperatures are unequal, $T_{g}=T_{d}+500 \mathrm{~K}$. A similar gaseous temperature enhancement is expected in the region in which the molecular emission is likely to arise. We estimate $S=0.1$ from Burke \& Hollenbach (1983).

The upper panel of Figure 2 gives the results for the hydrogen family at $R=1 \mathrm{AU}$. Three sets of abundance curves are plotted versus vertical column density for three levels of grain formation: no grain formation (dotted line); grain formation with $a_{\text {geom }}=7.07 \mu \mathrm{m}$ (dashed curve); grain formation with $a_{\text {geom }}=0.707 \mu \mathrm{m}$ (solid curve); they correspond respectively to models 1,3 , and 2 in Table 1 . Note that the usual interstellar value is $a_{g}=0.035 \mu \mathrm{m}$. In accord with the discussion in Section 2.1, the two choices of $a_{\text {geom }}$ correspond to reduction factors of 20 and 200 in the grain area per $\mathrm{H}$ nucleus compared to interstellar medium (ISM) values. If we define the location of the H-to- $\mathrm{H}_{2}$ transition by the vertical column where $x(\mathrm{H})=x\left(\mathrm{H}_{2}\right)$, then the transition occurs higher in the atmosphere (at smaller vertical columns) as the grain area increases. For the case $a_{\text {geom }}=0.707 \mu \mathrm{m}$, the $\mathrm{H}-\mathrm{H}_{2}$ transition, where $x(\mathrm{H})=x\left(\mathrm{H}_{2}\right)$, is shifted upward by a factor of 4 in vertical column from 
Table 2

Warm Molecular Columns

\begin{tabular}{|c|c|c|c|c|c|c|c|}
\hline \multicolumn{8}{|c|}{$R=0.25 \mathrm{AU}$} \\
\hline Model & $N_{\mathrm{H}}\left(\mathrm{cm}^{-2}\right)$ & $T(\mathrm{~K})$ & $x(\mathrm{e})$ & $x\left(\mathrm{H}_{2}\right)$ & $N\left(\mathrm{H}_{2} \mathrm{O}\right)\left(\mathrm{cm}^{-2}\right)$ & $N(\mathrm{CO})\left(\mathrm{cm}^{-2}\right)$ & $N(\mathrm{OH})\left(\mathrm{cm}^{-2}\right)$ \\
\hline $1^{\mathrm{a}}$ & $1.7(21)-9.5(21)$ & $300-560$ & $2.0(-6)-4.2(-6)$ & $5.5(-2)-4.5(-1)$ & $1.1(18)$ & $1.1(18)$ & $2.8(15)$ \\
\hline $2^{b}$ & $9.3(20)-9.5(21)$ & $300-810$ & $1.6(-6)-7.9(-6)$ & $7.3(-2)-4.9(-1)$ & $1.5(18)$ & $1.1(18)$ & $2.4(15)$ \\
\hline $3^{c}$ & $1.7(21)-1.4(22)$ & $310-650$ & $1.5(-6)-4.2(-6)$ & $4.1(-2)-4.9(-1)$ & $2.8(18)$ & $1.6(18)$ & $1.3(15)$ \\
\hline $4^{\mathrm{d}}$ & $9.3(20)-9.5(21)$ & $300-810$ & $1.5(-6)-7.9(-6)$ & $7.5(-2)-4.9(-1)$ & $1.6(18)$ & $1.1(18)$ & $2.4(15)$ \\
\hline $5^{\mathrm{e}}$ & $2.1(21)-1.9(22)$ & $300-720$ & $7.4(-7)-4.1(-6)$ & $4.7(-2)-4.9(-1)$ & $3.7(18)$ & $2.3(18)$ & $1.4(15)$ \\
\hline $6^{\mathrm{f}}$ & $1.4(21)-1.9(22)$ & $300-1000$ & $5.7(-7)-1.1(-5)$ & $3.4(-1)-4.9(-1)$ & $4.0(18)$ & $2.3(18)$ & $7.9(14)$ \\
\hline \multicolumn{8}{|c|}{$R=0.5 \mathrm{AU}$} \\
\hline Model & $N_{\mathrm{H}}\left(\mathrm{cm}^{-2}\right)$ & $T(\mathrm{~K})$ & $x(\mathrm{e})$ & $x\left(\mathrm{H}_{2}\right)$ & $N\left(\mathrm{H}_{2} \mathrm{O}\right)\left(\mathrm{cm}^{-2}\right)$ & $N(\mathrm{CO})\left(\mathrm{cm}^{-2}\right)$ & $N(\mathrm{OH})\left(\mathrm{cm}^{-2}\right)$ \\
\hline $1^{\mathrm{a}}$ & $1.2(21)-2.1(21)$ & $320-510$ & $4.7(-6)-5.7(-6)$ & $4.4(-2)-2.6(-1)$ & $6.2(15)$ & $1.0(17)$ & $7.3(14)$ \\
\hline $2^{b}$ & $4.3(20)-2.1(21)$ & $320-790$ & $5.1(-6)-9.2(-6)$ & $2.9(-2)-4.7(-1)$ & $3.0(17)$ & $2.0(17)$ & $1.7(15)$ \\
\hline $3^{c}$ & $9.2(20)-4.8(21)$ & $330-600$ & $2.0(-6)-3.5(-6)$ & $3.5(-2)-4.7(-1)$ & $7.9(17)$ & $4.9(17)$ & $1.0(15)$ \\
\hline $4^{\mathrm{d}}$ & $5.5(20)-2.1(21)$ & $320-790$ & $5.0(-6)-8.5(-6)$ & $3.1(-2)-4.7(-1)$ & $3.1(17)$ & $2.0(17)$ & $1.7(15)$ \\
\hline $5^{\mathrm{e}}$ & $1.9(21)-6.4(21)$ & $300-670$ & $1.6(-6)-2.8(-6)$ & $6.6(-2)-4.4(-1)$ & $9.2(17)$ & $6.5(17)$ & $8.8(14)$ \\
\hline $6^{\mathrm{f}}$ & $6.2(20)-5.5(21)$ & $320-1090$ & $1.5(-6)-1.1(-5)$ & $2.0(-1)-5.0(-1)$ & $1.1(18)$ & $5.6(17)$ & $7.9(14)$ \\
\hline \multicolumn{8}{|c|}{$R=1 \mathrm{AU}$} \\
\hline Model & $N_{\mathrm{H}}\left(\mathrm{cm}^{-2}\right)$ & $T(\mathrm{~K})$ & $x(\mathrm{e})$ & $x\left(\mathrm{H}_{2}\right)$ & $N\left(\mathrm{H}_{2} \mathrm{O}\right)\left(\mathrm{cm}^{-2}\right)$ & $N(\mathrm{CO})\left(\mathrm{cm}^{-2}\right)$ & $N(\mathrm{OH})\left(\mathrm{cm}^{-2}\right)$ \\
\hline$\overline{1^{\mathrm{a}}}$ & $>1.2(21)$ & $>300$ & $>1.2(-5)$ & $<7.5(-3)$ & $4.4(12)$ & $6.6(15)$ & $6.2(13)$ \\
\hline $2^{b}$ & $4.3(20)-1.2(21)$ & $330-770$ & $6.3(-6)-8.1(-6)$ & $9.7(-2)-4.6(-1)$ & $1.6(17)$ & $9.9(16)$ & $7.9(14)$ \\
\hline $3^{c}$ & $1.0(21)-2.9(21)$ & $310-560$ & $2.7(-6)-3.3(-6)$ & $6.4(-2)-4.5(-1)$ & $4.0(17)$ & $2.9(17)$ & $7.9(14)$ \\
\hline $4^{\mathrm{d}}$ & $4.3(20)-1.2(21)$ & $330-770$ & $6.2(-6)-8.1(-6)$ & $9.9(-2)-4.6(-1)$ & $1.6(17)$ & $9.2(16)$ & $6.6(14)$ \\
\hline $5^{\mathrm{e}}$ & $1.8(21)-3.8(21)$ & $340-630$ & $1.6(-6)-2.1(-6)$ & $7.4(-2)-3.7(-1)$ & $4.5(17)$ & $3.7(17)$ & $5.1(14)$ \\
\hline $6^{\mathrm{f}}$ & $1.0(21)-3.8(21)$ & $300-1020$ & $1.6(-6)-8.0(-6)$ & $4.6(-1)-5.0(-1)$ & $6.5(17)$ & $3.3(17)$ & $1.6(14)$ \\
\hline \multicolumn{8}{|c|}{$R=2 \mathrm{AU}$} \\
\hline Model & $N_{\mathrm{H}}\left(\mathrm{cm}^{-2}\right)$ & $T(\mathrm{~K})$ & $x(\mathrm{e})$ & $x\left(\mathrm{H}_{2}\right)$ & $N\left(\mathrm{H}_{2} \mathrm{O}\right)\left(\mathrm{cm}^{-2}\right)$ & $N(\mathrm{CO})\left(\mathrm{cm}^{-2}\right)$ & $N(\mathrm{OH})\left(\mathrm{cm}^{-2}\right)$ \\
\hline $1^{\mathrm{a}}$ & $>9.4(20)$ & $>300$ & $>3.7(-5)$ & $<1.1(-3)$ & $3.0(11)$ & $5.0(13)$ & $3.2(13)$ \\
\hline $2^{b}$ & $3.9(20)-9.4(20)$ & $300-720$ & $6.6(-6)-8.6(-6)$ & $1.4(-1)-4.4(-1)$ & $1.0(17)$ & $8.4(16)$ & $1.1(15)$ \\
\hline $3^{c}$ & $9.4(20)-1.8(21)$ & $310-500$ & $3.4(-6)-3.7(-6)$ & $1.0(-1)-4.1(-1)$ & $1.7(17)$ & $1.7(17)$ & $6.2(14)$ \\
\hline $4^{\mathrm{d}}$ & $3.9(20)-9.4(20)$ & $300-720$ & $6.6(-6)-8.6(-6)$ & $1.4(-1)-4.5(-1)$ & $1.0(17)$ & $8.4(16)$ & $1.2(15)$ \\
\hline $5^{e}$ & $1.8(21)-3.4(21)$ & $320-600$ & $1.4(-6)-1.9(-6)$ & $9.8(-3)-3.4(-1)$ & $3.0(17)$ & $3.2(16)$ & $6.2(14)$ \\
\hline $6^{\mathrm{f}}$ & $4.5(20)-2.9(21)$ & $310-1170$ & $1.7(-6)-1.1(-5)$ & $3.0(-1)-5.0(-1)$ & $5.7(17)$ & $2.8(17)$ & $4.4(14)$ \\
\hline
\end{tabular}

Notes.

${ }^{\mathrm{a}}$ Gas phase only, $a_{\text {geom }}=0.707 \mu \mathrm{m}, \alpha=0.01$.

${ }^{\mathrm{b}} \mathrm{H}_{2}$ formation on grains, $a_{\mathrm{geom}}=0.707 \mu \mathrm{m}, \alpha=0.01$.

${ }^{\mathrm{c}} \mathrm{H}_{2}$ formation on grains, $a_{\text {geom }}=7.07 \mu \mathrm{m}, \alpha=0.01$.

${ }^{\mathrm{d}} \mathrm{H}_{2}$ and $\mathrm{H}_{2} \mathrm{O}$ formation on grains, $a_{\text {geom }}=0.707 \mu \mathrm{m}, \alpha=0.01$.

e Gas phase only, $a_{\text {geom }}=0.707 \mu \mathrm{m}, \alpha=1.0$.

${ }^{\mathrm{f}} \mathrm{H}_{2}$ formation on grains, $a_{\text {geom }}=0.707 \mu \mathrm{m}, \alpha=1.0$.

$N_{\mathrm{H}}=2.4 \times 10^{21} \mathrm{~cm}^{-2}$ to $N_{\mathrm{H}}=6 \times 10^{20} \mathrm{~cm}^{-2}$. The bottom panel of Figure 2 plots the temperature for the three cases in the upper panel. The dotted and solid temperature curves are roughly the same because they have the same grain area, i.e., $a_{\text {geom }}=0.707 \mu \mathrm{m}$. For the dashed curve, $a_{\text {geom }}=7.07 \mu \mathrm{m}$, the temperature is raised above the dust temperature because the gas and dust become less well coupled thermally as the grain surface area decreases. Comparing the upper with the lower panel of Figure 2, we see that the $\mathrm{H}-\mathrm{H}_{2}$ transition for the solid curves $\left(a_{\text {geom }}=0.707 \mu \mathrm{m}\right)$ occurs roughly in the middle of the transition region and that this model yields a significant column of warm water $(T>300 \mathrm{~K}), N\left(\mathrm{H}_{2} \mathrm{O}\right) \approx 2 \times 10^{17} \mathrm{~cm}^{-2}$.

Figure 2 shows that grain formation of $\mathrm{H}_{2}$ is important in the warm transition region of the disk atmosphere. Increasing the abundance of $\mathrm{H}_{2}$ also increases the abundances of $\mathrm{OH}$ and $\mathrm{H}_{2} \mathrm{O}$, which leads to a decrease in the abundance of $\mathrm{H}^{+}$through the reactions in Equations (8) and (9). This adds a degree of runaway to the abundances since decreasing $\mathrm{H}^{+}$increases the amounts of $\mathrm{OH}$ and $\mathrm{H}_{2} \mathrm{O}$. The two main oxygen species, $\mathrm{O}$ and $\mathrm{H}_{2} \mathrm{O}$, are plotted in the middle panel of Figure 2. The increased abundance of $\mathrm{H}_{2}$ manifests itself in a significant enhancement of $\mathrm{H}_{2} \mathrm{O}$ in the warm transition region. Without grain formation of $\mathrm{H}_{2}$, full conversion of $\mathrm{O}$ to $\mathrm{H}_{2} \mathrm{O}$ does not occur until a vertical column $N_{\mathrm{H}}=1.5 \times 10^{23} \mathrm{~cm}^{-2}$ has been reached, at which depth dust cooling has overpowered gas heating and the gas and dust temperatures are the same. At $R=1 \mathrm{AU}$, this temperature is of order $100 \mathrm{~K}$, so such a model cannot account for the warm water observed at NIR and MIR wavelengths. However, with grain formation of $\mathrm{H}_{2}$, full conversion occurs higher in the atmosphere where the gas temperature is elevated above that of the dust. For example, for the case $a_{\text {geom }}=7.07 \mu \mathrm{m}$, the $\mathrm{O}-\mathrm{H}_{2} \mathrm{O}$ transition occurs near $N_{\mathrm{H}}=10^{21} \mathrm{~cm}^{-2}$ and, for $a_{\text {geom }}=0.707 \mu \mathrm{m}$, it occurs at $N_{\mathrm{H}}=4 \times 10^{20} \mathrm{~cm}^{-2}$. In both cases, there is a warm layer where almost all the available oxygen is in water (recall that $x_{\mathrm{O}}=3.5 \times 10^{-4}$ and $x_{\mathrm{C}}=1.4 \times 10^{-4}$ ). For the case $a_{\text {geom }}=0.707 \mu \mathrm{m}$, this layer contains a water column, $N\left(\mathrm{H}_{2} \mathrm{O}\right) \approx 2 \times 10^{17} \mathrm{~cm}^{-2}$, and for $a_{\text {geom }}=7.07 \mu \mathrm{m}$, the water column is $N\left(\mathrm{H}_{2} \mathrm{O}\right) \approx 4 \times 10^{17} \mathrm{~cm}^{-2}$. This increase occurs because the increase in $a_{\text {geom }}$ implies a decrease in dust cooling, which results in a shift downward of the warm region into denser 


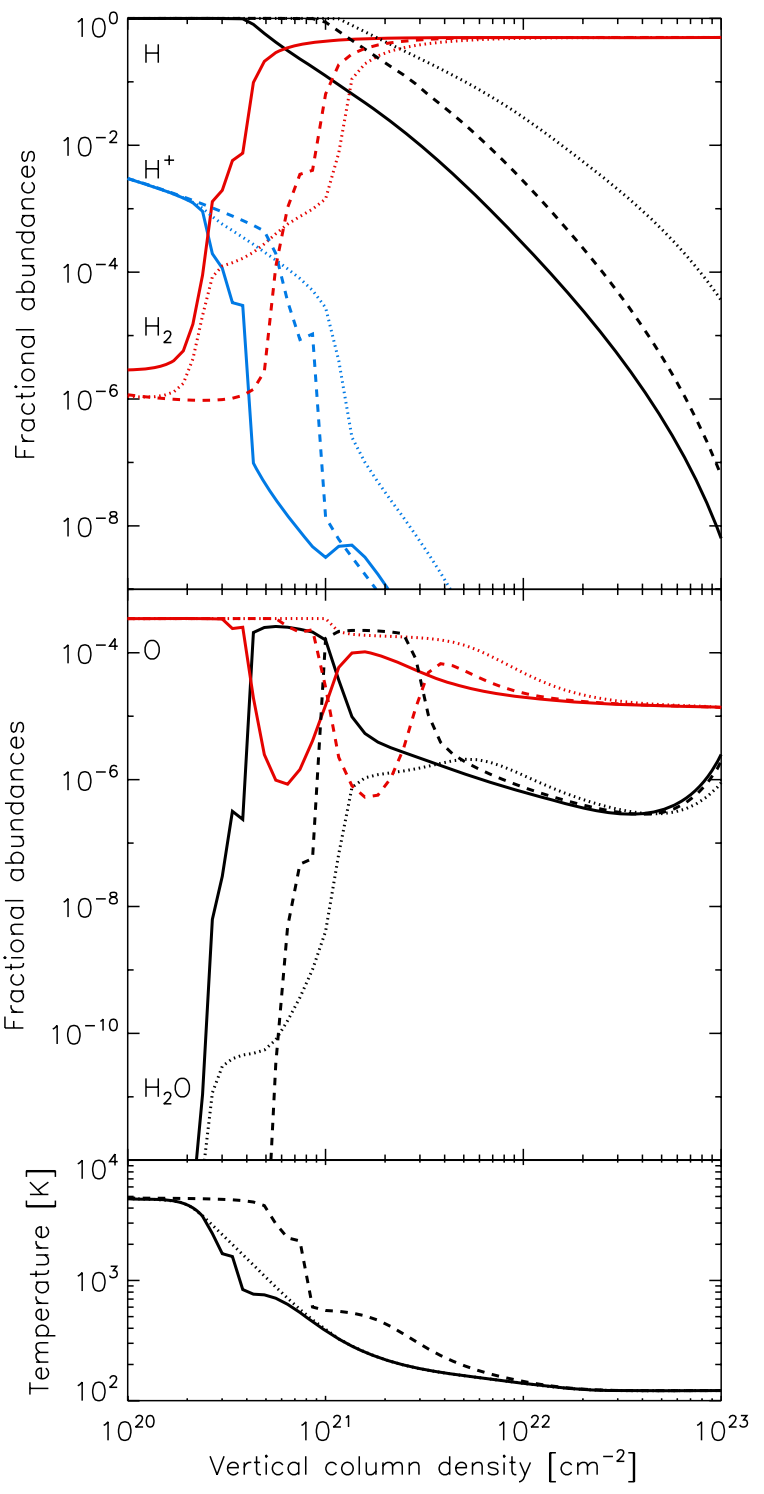

Figure 2. Main hydrogenic species, atomic oxygen and water, and gas temperature at 1 AU plotted vs. vertical column density for three cases of grain formation: no grain formation (dotted line); grain formation with $a_{\text {geom }}=7.07 \mu \mathrm{m}$ (dashed curve); grain formation with $a_{\text {geom }}=0.707 \mu \mathrm{m}$ (solid curve). These three cases correspond respectively to models 1,3 , and 2 in Tables 1 and 2 .

(A color version of this figure is available in the online journal.)

gas. These columns of warm water approach the values obtained from recent Spitzer observations assuming thermal populations, $\gtrsim 10^{17} \mathrm{~cm}^{-2}$ (Carr \& Najita 2008; Salyk et al. 2008). The $\mathrm{OH}$ abundance also rises as the transition to water is approached, reaching abundance levels $\sim 10^{-7}$ to $10^{-6}$ and then decreasing rapidly with increasing vertical column of hydrogen.

Below these layers of near-maximum water abundance high in the atmosphere, the water abundance drops two orders of magnitude before recovering at very large depths. The origin of this abundance swing can be found in the approximate formula Equation (10). Once $\mathrm{H}_{2}$ has been fully formed, the $\mathrm{H}_{2} \mathrm{O}$-to-O ratio is governed by the relative importance of the temperature decline, as the gas and dust temperatures become more and more closely coupled, and by the decrease in the abundances of the ions $\mathrm{H}^{+}$and $\mathrm{He}^{+}$which destroy $\mathrm{H}_{2} \mathrm{O}$. With increasing depth, the decrease in temperature first reduces the production

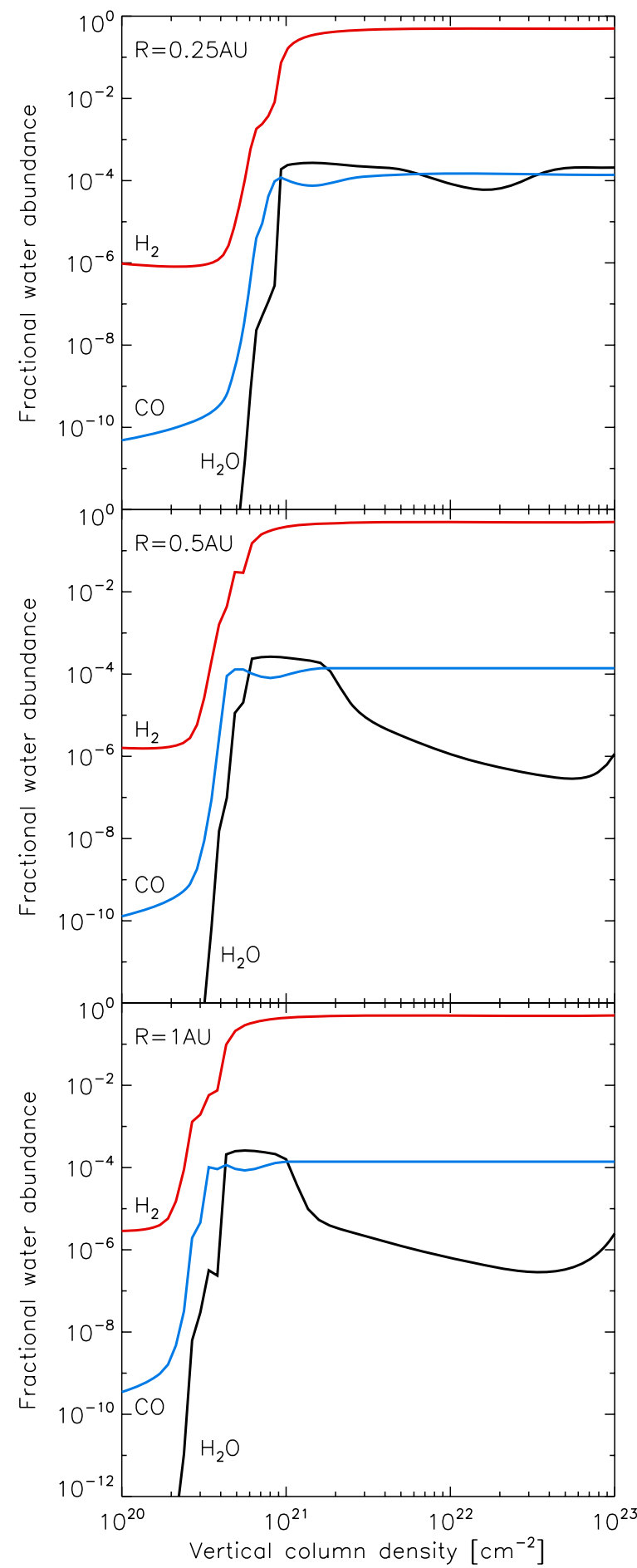

Figure 3. $\mathrm{H}_{2}, \mathrm{CO}$, and water abundances at $R=0.25$ (top), 0.5 (middle), and 1.0 (bottom) AU for model 2 with $\mathrm{H}_{2}$ formation on grains and $a_{\mathrm{geom}}=0.707 \mu \mathrm{m}$. (A color version of this figure is available in the online journal.)

of water, but then the attenuated X-ray flux (and consequent low ion abundance) removes any effective destruction so that its abundance increases again.

The full conversion to water is closely associated with the two other chemical transitions, $\mathrm{H}$ to $\mathrm{H}_{2}$ and $\mathrm{C}$ to $\mathrm{CO}$. These transitions are displayed in Figure 3 at two smaller radii, $R=0.25$ and $R=0.5 \mathrm{AU}$, as well as at $R=1.0 \mathrm{AU}$ (used in the previous figures). In all cases, the transitions occur in 
sequence at increasing vertical columns: first $\mathrm{H}$ to $\mathrm{H}_{2}$ at the low abundance of $\mathrm{H}_{2}\left(\sim 10^{-6}\right)$ needed to start the warm radical reaction sequence described in Section 2.1; then $\mathrm{C}$ to $\mathrm{CO}$, next $\mathrm{O}$ to $\mathrm{H}_{2} \mathrm{O}$, and finally full conversion of $\mathrm{H}$ to $\mathrm{H}_{2}$. Full conversion of the residual $\mathrm{O}$ to $\mathrm{H}_{2} \mathrm{O}$ occurs where $x\left(\mathrm{H}_{2}\right) \sim 0.1$.

We conclude that the formation of $\mathrm{H}_{2}$ on dust grains can play a key role in the synthesis of water in protoplanetary disk atmospheres. The efficiency of grain formation is determined overall by the effective surface area of the grains, which we have expressed in terms of the geometric mean $a_{\text {geom }}$ of the minimum and maximum grain sizes in a MRN distribution. Both $a_{\text {geom }}$ and the efficiency of grain formation are affected by the amount of grain growth and settling. Figure 2 gives a concrete illustration of this effect at $1 \mathrm{AU}$. The top panel of Figure 2 shows that increasing $a_{\text {geom }}$ from $0.707 \mu \mathrm{m}$ to $7.07 \mu \mathrm{m}$ shifts the $\mathrm{H}-\mathrm{H}_{2}$ transition downward (to larger values of $N_{\mathrm{H}}$ ). This means that the layer where the water abundance is a maximum is also shifted downward, as shown in the dashed curves in the middle panel of Figure 2.

We have focused on two aspects of grain formation of $\mathrm{H}_{2}$ that are important in disks, the extension of the standard theory to warm dust temperatures and the effect of grain growth and settling on the effective grain surface area. For the latter we use a single average parameter, the geometric mean of the minimum and maximum grain size $\left(a_{\text {geom }}\right)$, ignoring many complexities such as the nature of the grain surface and the variation of dust properties with grain size. We also assume that the same grain area applies to both the formation of $\mathrm{H}_{2}$ and to the thermal coupling of the gas and the dust. The issue of $\mathrm{H}_{2}$ formation on warm grains arose earlier in connection with Infrared Space Observatory measurements of the pure rotational transitions of $\mathrm{H}_{2}$ in photon-dominated regions (PDRs) that seemed to require higher temperatures or more $\mathrm{H}_{2}$ than predicted by PDR models (e.g., van Dishoeck 2004). Habart et al. (2004) confronted this problem by considering the $\mathrm{H}_{2}$ grain formation rate as a variable in fitting measured 0-0 S(3) to 1-0 S(1) line ratios with a PDR model. They found "normal" (diffuse cloud) rate coefficients for high-excitation regions like the Orion bar and values enhanced by a factor of 5 for moderate excitation PDRs. They also determined a rough scaling between the $\mathrm{H}_{2}$ 1-0 S(1) line and the strength of PAH features that suggested $\mathrm{H}_{2}$ formation on PAHS might be responsible for the empirically determined formation rates. Habart et al. (2004) and Cazaux et al. (2006) showed that "indirect" chemisorption, where a second $\mathrm{H}$ atom reaches a chemisorption site from a physisorption site, could explain the empirical grain formation rates deduced for PDRs if the barrier between the two kinds of sites is small enough $(\sim 0.05 \mathrm{eV})$ and if a significant population of small grains is present. While we do not consider $\mathrm{H}_{2}$ formation by PAHs here because there are likely to be few PAHs in the inner regions of protoplanetary disks (see the Appendix), we do employ a reduced population of small grains for the synthesis of $\mathrm{H}_{2}$.

The question of whether a barrier as small a $0.05 \mathrm{eV}$ between physisorption and chemisorption sites is appropriate may have been resolved by laboratory experiments with graphite at moderate temperatures which suggest that the formation of $\mathrm{H}$-atom dimers (Hornekær et al. 2006a) or clusters (Hornekær et al. 2006b) may be an important step in grain formation of $\mathrm{H}_{2}$. Although the Cazaux-Tielens theory of grain formation of $\mathrm{H}_{2}$ yields a consistent picture for PDRs, it may not carry over to protoplanetary disk atmospheres of disks. The densities are much higher and the abundance of small grains is much less than in PDRs. However, the demonstrated sensitivity of molecular

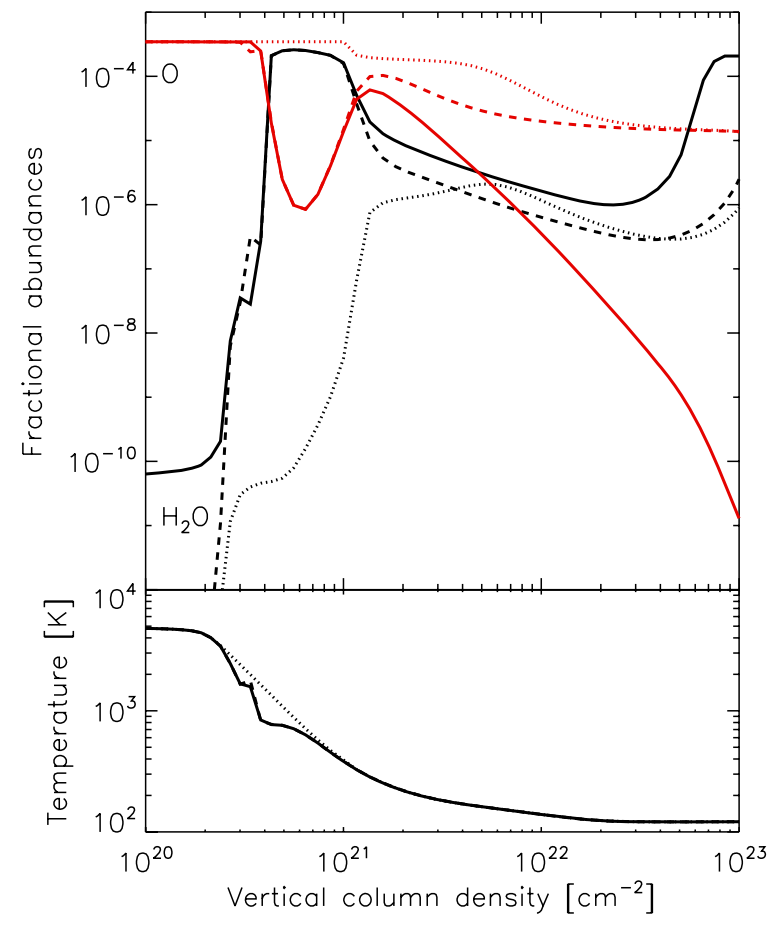

Figure 4. Oxygen species and temperature at $1 \mathrm{AU}$ vs. vertical column density for three cases of $\mathrm{H}_{2}$ and $\mathrm{H}_{2} \mathrm{O}$ formation on grains: no grain formation (dotted line); $\mathrm{H}_{2}$ formation on grains with $a_{\text {geom }}=0.707 \mu \mathrm{m}$ (dashed curve) $\mathrm{H}_{2}$ and $\mathrm{H}_{2} \mathrm{O}$ formation on grains with $a_{\text {geom }}=0.707 \mu \mathrm{m}$ (solid curve). These three cases correspond respectively to models 1,3 , and 4 in Tables 1 and 2 .

(A color version of this figure is available in the online journal.)

abundances to the abundance of $\mathrm{H}_{2}$ may eventually lead to a better understanding of $\mathrm{H}_{2}$ formation in disks when more extensive observations of species like water become available.

\subsection{The Formation of Water on Dust Grains}

Water may also be formed by the sticking of $\mathrm{O}$ atoms and $\mathrm{OH}$ radicals on warm grains and subsequent reactions with adsorbed hydrogen atoms or other species. In regions where water is not already the dominant oxygen species, atomic $\mathrm{O}$ is much more abundant than $\mathrm{OH}$, and we consider only this case. For this purpose, we adapt the formula for $\mathrm{H}_{2}$ formation on grains (Equation (15)) by dropping the $1 / 2$ factor, noticing that $v(\mathrm{O})=(1 / 4) v(\mathrm{H})$ and introducing a grain formation efficiency factor $\epsilon(\mathrm{O})$ and a sticking factor $S(\mathrm{O})$,

$$
\begin{aligned}
R\left(\mathrm{H}_{2} \mathrm{O}\right)= & 2 \times 10^{-19} \mathrm{~cm}^{3} \mathrm{~s}^{-1} T_{\mathrm{g}}^{1 / 2} n(\mathrm{O}) n_{\mathrm{H}} \\
& \times\left(\frac{\rho_{\mathrm{d}} / \rho_{\mathrm{g}}}{0.01}\right)\left(\frac{\mu \mathrm{m}}{a_{g}}\right) \epsilon(\mathrm{O}) S(\mathrm{O}) .
\end{aligned}
$$

We have implemented Equation (16) using $\epsilon(\mathrm{O})=1$ and $S(\mathrm{O})=1$ to estimate the maximum role of water formation on grains. Figure 4 displays the changes in $\mathrm{O}$ and $\mathrm{H}_{2} \mathrm{O}$ for the case of no grain formation, i.e., pure gas-phase synthesis (dotted curve), grain formation of only $\mathrm{H}_{2}$ with $a_{\text {geom }}=7.07 \mu \mathrm{m}$ (dashed curve), and grain formation of both $\mathrm{H}_{2}$ and $\mathrm{H}_{2} \mathrm{O}$ with $a_{\text {geom }}=0.707 \mu \mathrm{m}$ (solid curve). These cases correspond respectively to models 1,3 , and 4 in Tables 1 and 2 . This model of maximal grain formation leads to two effects for $R=1 \mathrm{AU}$. At very small columns, $N_{\mathrm{H}}<2 \times 10^{20} \mathrm{~cm}^{-2}$, the water abundance is increased by 3 dex to the $10^{-10}$ level, but this level of hot water is probably not observable. Grain formation of water 
also plays some role deeper down $\left(N_{\mathrm{H}}>10^{21} \mathrm{~cm}^{-2}\right)$ where gas-phase synthesis is shut off by the low temperature. For $N_{\mathrm{H}}>2 \times 10^{21} \mathrm{~cm}^{-2}$ at $1 \mathrm{AU}$, the increased abundance of water should hardly affect the amount of observable warm water since it occurs where the temperature is close to $100 \mathrm{~K}$. However, inside of $0.5 \mathrm{AU}$, water at large depths $N_{\mathrm{H}}>2 \times 10^{21} \mathrm{~cm}^{-2}$ will be observable because the gas is much warmer there. Nonetheless, the top layer of high-abundance water is the most important, and we conclude that grain formation of water is unlikely to play a dominant role in the inner disk.

Another process that might contribute to the formation of water in the dense inner regions of a protoplanetary disk is radiative association,

$$
\mathrm{O}+\mathrm{H}_{2} \rightarrow \mathrm{H}_{2} \mathrm{O}+h v .
$$

To the best of our knowledge, this reaction has not been discussed in the literature. To exceed the production of water by grain formation, the rate coefficient for radiative association would have to be larger than $10^{-17} \mathrm{~cm}^{3} \mathrm{~s}^{-1}$. Although this is a typical rate coefficient for the radiative association of neutral species, it may not apply to water, at least without detailed consideration of the relevant potential energy curves. The rate coefficient for the radiative association of $\mathrm{OH}$ and H has been calculated by Fields et al. (1980). It is a strongly decreasing function of temperature, large at low temperatures $\left(\gtrsim 10^{-14} \mathrm{~cm}^{3} \mathrm{~s}^{-1}\right)$ but small in warm regions $\left(\lesssim 10^{-18} \mathrm{~cm}^{3} \mathrm{~s}^{-1}\right.$ above $300 \mathrm{~K}$ ). This process might be relevant for cool atomic $\mathrm{H}$ regions, but not in the atmospheres of the inner disks under consideration here. On the other hand, if the rate coefficient for Equation (17) is significantly larger than $10^{-17} \mathrm{~cm}^{3} \mathrm{~s}^{-1}$ at moderate temperatures, this reaction would be an important source of water.

\subsection{Mechanical Heating}

Finally we consider nonradiative heating processes that can affect the abundance and location of water. To illustrate this possibility, we adopt the mechanical heating model used in GNI04,

$$
\Gamma_{\mathrm{acc}}=\frac{9}{4} \alpha_{\mathrm{h}} \rho_{\mathrm{g}} c^{2} \Omega
$$

where $\rho_{\mathrm{g}}$ is the local gas mass density, $c$ is the isothermal sound speed, $\Omega$ is the angular rotation speed, and $\alpha_{\mathrm{h}}$ is a phenomenological constant. GNI04 used values in the range $\alpha_{\mathrm{h}}=0.01-1.0$; they showed that the value 0.01 differs negligibly from the case $\alpha_{\mathrm{h}}=0$ for pure X-ray heating. Mechanical heating might arise from viscous accretion generated by the MRI or from the interaction of a stellar wind with the upper layers of the disk. The change from the dotted to the dashed curves in Figure 5 shows the effect of increasing $\alpha_{h}$ from 0.01 to 1.0 for the case of no formation of $\mathrm{H}_{2}$ on grains. As discussed in GNI04, mechanical heating causes the transition from high to low temperatures to start deeper down in the disk, near $N_{\mathrm{H}}=10^{21} \mathrm{~cm}^{-2}$. It also produces a region of warm water $(T>300 \mathrm{~K})$ at high abundance. The solid curve in Figure 5 shows that the combination of mechanical heating and grain formation of $\mathrm{H}_{2}$ can increase the thickness of warm water before gas-phase production is cut off at low temperatures. The column of warm water for model 6 is now $N\left(\mathrm{H}_{2} \mathrm{O}\right)=6.5 \times 10^{17} \mathrm{~cm}^{-2}$ at $1 \mathrm{AU}$, and even larger at smaller radii. These columns are of the same order as reported by Carr \& Najita (2008) and Salyk et al. (2008). This result illustrates how mechanical heating can be important in determining the abundance of water and highlights the need for further studies of mechanical heating processes.

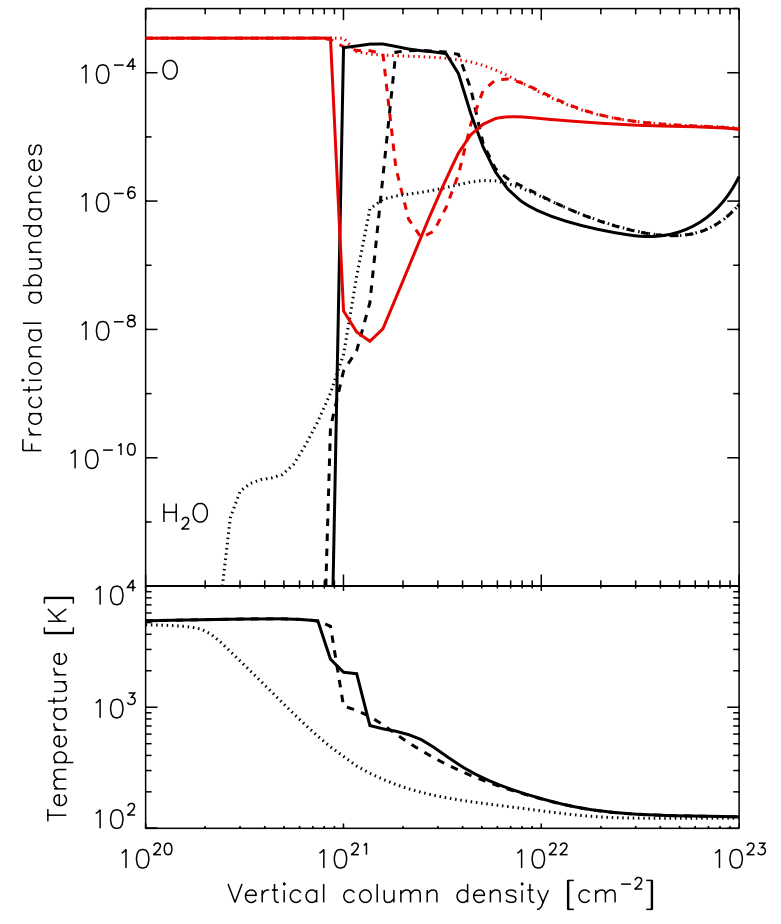

Figure 5. Effect of mechanical heating on the abundance of molecular hydrogen and water: $\alpha_{\mathrm{h}}=0.01$ and no grain formation (dotted curve); $\alpha_{\mathrm{h}}=1.0$ and no grain formation (dashed curve); $\alpha_{\mathrm{h}}=1.0$ with grain formation of $\mathrm{H}_{2}$ and $a_{\text {geom }}=0.707 \mu \mathrm{m}$ (solid curve). These three cases correspond respectively to models 1, 5, and 6 in Tables 1 and 2 .

(A color version of this figure is available in the online journal.)

\section{DISCUSSION}

In the previous section, we addressed the question of what determines observable levels of water in the inner region of a protoplanetary disk. One critical factor is the synthesis of $\mathrm{H}_{2}$, which is required for the production of $\mathrm{OH}$ and $\mathrm{H}_{2} \mathrm{O}$ from atomic $\mathrm{O}$ via neutral radical reactions. The gas must be warm in order for gas-phase synthesis to proceed, and the synthesis of $\mathrm{H}_{2}$ must occur at moderate depths into the transition region in order for the $\mathrm{H}^{+}$abundance to be reduced by the attenuation of the X-rays and by the increase in density. All of these factors, the $\mathrm{H}_{2}$ abundance, the temperature, and the level of ionization, can come together to produce maximum abundances of water in the thermal-chemical transition region that lies between the hot upper layer and the midplane of the atmosphere of an X-ray irradiated protoplanetary disk.

We arrived at this conclusion by showing how sensitive the amount of warm water in protoplanetary disk atmospheres is to poorly known processes involving the $\mathrm{H}_{2}$ molecule. We used the device of comparing the $\mathrm{H}_{2}$ and $\mathrm{H}_{2} \mathrm{O}$ abundances in the original model of GNI04 (corrected for the error in the rate coefficient $k_{5}$ in Equation (5)) with the abundances calculated for new rate coefficients and processes. These include (1) the adoption of the Flower \& Harris (2007) rate coefficients for the three-body reactions, Equation (3); (2) the introduction of grain formation of $\mathrm{H}_{2}$ on the basis of the theory of Cazaux \& Tielens (2002, 2004), Equation (15); (3) the assumption that $\mathrm{H}_{2} \mathrm{O}$ is formed on grains at the maximum rate, Equation (16); and (4) mechanical heating of the atmosphere, Equation (18). These changes were illustrated by the abundance patterns in the previous figures. Although we emphasized water, the changes we have discussed also affect atomic $\mathrm{O}$ and $\mathrm{OH}$ and the species with which they interact. 
An important measure of the changes is the column density of warm water, defined to have $T>300 \mathrm{~K}$ and an abundance large enough to potentially make a significant contribution to observable line fluxes. In practice, this column begins where the water abundance first achieves its maximum value and ends where the temperature drops below about $300 \mathrm{~K}$. These columns are given in Table 2 for the models considered in the previous section, along with the columns of warm $\mathrm{OH}$ and $\mathrm{CO}$. The table includes the corresponding ranges in hydrogen column density $\left(N_{\mathrm{H}}\right)$, temperature $(T)$, electron fraction $\left(x_{\mathrm{e}}\right)$, and molecular hydrogen abundance $\left(x\left(\mathrm{H}_{2}\right)\right)$. Not only do these abundances express the physical conditions where the warm water occurs, but they also determine which collision partners might be important in exciting observable transitions. Excitation of the low-lying rotational and rovibrational levels of water by electrons is typically $10^{4}$ times stronger than for $\mathrm{H}_{2}$ (Faure \& Josselin 2008). Thus, electronic excitation will not dominate in these warm water regions. But the $\mathrm{H}-\mathrm{H}_{2}$ transition may not be complete, so that atomic $\mathrm{H}$ collisional excitation is likely to play a role, not just $\mathrm{H}_{2}$ collisions.

Table 2 contains information about the radial variation of the warm molecular columns of $\mathrm{H}_{2} \mathrm{O}, \mathrm{OH}$, and $\mathrm{CO}$. The warm water column $N\left(\mathrm{H}_{2} \mathrm{O}\right)$ rapidly increases with decreasing radius, faster than $1 / R$ for $R \lesssim 0.5 \mathrm{AU}$. The inner disk gets warmer due to increased accretion heating, which reduces the cooling of the gas by the dust. All of the models yield $N\left(\mathrm{H}_{2} \mathrm{O}\right)>10^{18} \mathrm{~cm}^{-2}$ for small radii $(R \lesssim 0.5 \mathrm{AU})$. This result opens up the possibility that the water lines may have broad wings for inclined disks. Detailed excitation calculations are needed to deal with this issue, not just for water but for other molecules for which line shape measurements are feasible, especially CO. Accretion heating becomes increasingly important with decreasing $R$, to the point where pure gas-phase processes (model 5 in Table 1) give about the same water columns as model 6 (with $\mathrm{H}_{2}$ grain formation). This can be seen in detail in Figure 6 which shows water abundance profiles at several radii for pure gas-phase chemistry.

The results of the few reported observations of water in disks around young low-mass stars (Carr et al. 2004; Carr \& Najita 2008; Salyk et al. 2008) have been expressed in terms of a column density and an excitation temperature. For the typical T Tauri star AA Tau, Carr and Najita found $N\left(\mathrm{H}_{2} \mathrm{O}\right)=6.5 \times 10^{17} \mathrm{~cm}^{-2}$ and a characteristic excitation temperature of $\sim 575 \mathrm{~K}$. The same quantities for $\mathrm{OH}$ and $\mathrm{CO}$ are $N(\mathrm{OH})=8.1 \times 10^{16} \mathrm{~cm}^{-2}$ (characteristic temperature of $\sim 525 \mathrm{~K}$ ) and $N(\mathrm{CO})=4.9 \times 10^{17} \mathrm{~cm}^{-2}$ (characteristic temperature of $\sim 900 \mathrm{~K}$ ). Salyk et al. (2008) reported similar water columns for two other T Tauri stars. These column densities have been derived assuming thermal populations, so they may be underestimates or overestimates, depending on the excitation conditions. Although the model calculations in this paper were done primarily to illustrate how certain physical and chemical processes affect the amount of observable water in protoplanetary disks - and not to model any particular disk, it is of some interest to compare the observations with the results in Table 2. The presumed improvements in the treatment of the formation of $\mathrm{H}_{2}$, especially grain formation, bring the calculated column of warm water close to the observed range $N\left(\mathrm{H}_{2} \mathrm{O}\right)>10^{17}-10^{18} \mathrm{~cm}^{-2}$. If the heating of the atmosphere is increased, the reported columns $N\left(\mathrm{H}_{2} \mathrm{O}\right)=(6-8) \times 10^{17} \mathrm{~cm}^{-2}$ can be reached or even exceeded. In drawing this conclusion, we have not varied the density distribution or the X-ray irradiation, which can affect the water column. For example, the

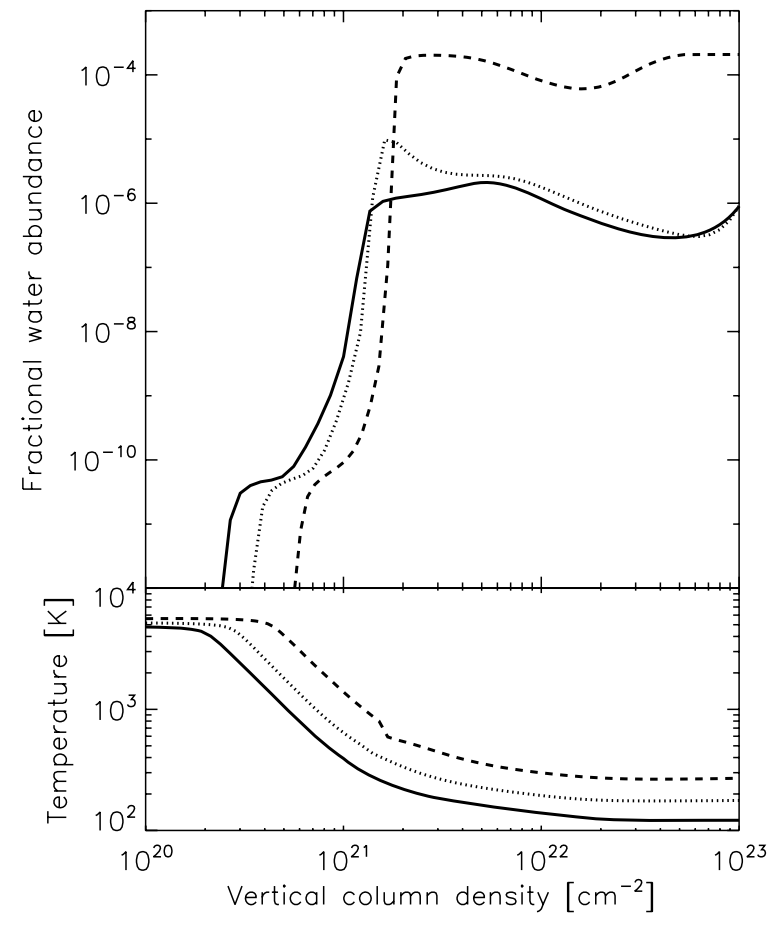

Figure 6. Water abundance and temperature profiles at $R=0.25$ (dashed), 0.5 (dotted), and 1.0 (solid) AU for model 1 for the case of only gas-phase reactions (model 1) and $a_{\text {geom }}=0.707 \mu \mathrm{m}$.

X-ray emission of AA Tau appears to be typical for an active classical T Tauri star (Grosso et al. 2007), with a minimum or quiescent X-ray luminosity $L_{\mathrm{x}}=10^{30} \mathrm{erg} \mathrm{s}^{-1}$ and temperature $T_{\mathrm{X}}=2.5 \mathrm{keV}$. Both parameters are somewhat different than used here based on the GNI model, $L_{\mathrm{x}}=2 \times 10^{30} \mathrm{erg} \mathrm{s}^{-1}$ and temperature $T_{\mathrm{X}}=1.0 \mathrm{keV}$. In addition to the effects of choices in model parameters, the predictions in Table 2 are subject to the limitations of our demonstration model (GNI04), e.g., the deficiencies in the thermal model and specifically the neglect of water heating and cooling, as discussed in Section 2.1.

One feature of the model calculations is that the column density of $\mathrm{OH}$ is much less than that of water. According to the last column of Table 2, the typical column density of warm $\mathrm{OH}$ in the inner disk is, to within a factor of $2, N(\mathrm{OH}) \sim 10^{15} \mathrm{~cm}^{-2}$. The ratio of the warm $\mathrm{H}_{2} \mathrm{O}$ to the warm $\mathrm{OH}$ column density ranges from a few hundred to several thousand, due mainly to the changes in the chemical model. The measured warm $\mathrm{OH}$ column for AA Tau is $N(\mathrm{OH})=8 \times 10^{16} \mathrm{~cm}^{-2}$, and none of the entries in Table 2 approach this value. This points to a deficiency in our calculation of the $\mathrm{OH}$ abundance. Staying within the confines of gas-phase chemistry, this problem might be solved by including FUV photodissociation of $\mathrm{OH}$ and $\mathrm{H}_{2} \mathrm{O}$, taking into account that the cross section for water is several times larger than that for $\mathrm{OH}$. Preferential photodissociation of water might then lead to a significant increase in the amount of $\mathrm{OH}$. An extreme example of this effect is the detection in the $L$ band $(3 \mu \mathrm{m})$ of the lines of $\mathrm{OH}$ but not $\mathrm{H}_{2} \mathrm{O}$ in the disks around two Herbig Ae stars (Mandell et al. 2008). In this case, there is ample FUV from the A star to destroy circumstellar water in favor of $\mathrm{OH}$. For T Tauri star disks, however, the stellar FUV flux is much smaller, and it may be unable to shift the balance from $\mathrm{H}_{2} \mathrm{O}$ to $\mathrm{OH}$, especially in the presence of neutral gas phase synthesis reactions which tend to drive $\mathrm{OH}$ to $\mathrm{H}_{2} \mathrm{O}$. This is the situation in our model, despite the fact that the rate 
coefficient for the reaction that destroys water, Equation (9) $\left(\mathrm{H}^{+}+\mathrm{H}_{2} \mathrm{O} \rightarrow \mathrm{H}_{2} \mathrm{O}^{+}+\mathrm{H}\right.$; Anicich 1993), is four times larger than that for $\mathrm{OH}$, Equation $(8)\left(\mathrm{H}^{+}+\mathrm{OH} \rightarrow \mathrm{OH}^{+}++\mathrm{H}\right.$; UMIST guess, Woodall et al. 2007). However, Bergin et al. (2003) have pointed out that the photodissociation of water is sensitive to the spectral shape of the stellar FUV, in particular to the strength of the Ly $\alpha$ line, which dominates the FUV emission in some T Tauri stars such as TW Hya. Thus, sources with stronger Ly $\alpha$ emission would be more effective in destroying water. On the other hand, $\mathrm{OH}$ can also be destroyed by Ly $\alpha$ radiation (van Dishoeck \& Dalgarno 1983). The rates of direct destruction of $\mathrm{OH}$ and $\mathrm{H}_{2} \mathrm{O}$ by X-rays are about the same, since both cases depend largely on $K$-shell absorption by a single oxygen atom.

Going beyond pure gas-phase chemistry, $\mathrm{OH}$ may be formed by desorption from icy grain mantles transported from cold regions to the warm source of the observed MIR lines within an $\mathrm{AU}$ or so from the star. The release could proceed by either thermal or photodesorption (Ciesla \& Cuzzi 2006). The latter process has been suggested to be the origin of the highly excited $\mathrm{OH}$ ground rotational lines seen in HH 211 (Tappe et al. 2008). In this case, the FUV radiation is shock generated, and it can desorb both $\mathrm{OH}$ and $\mathrm{H}_{2} \mathrm{O}$ (e.g., Andersson et al. 2008). When the water is photodissociated, $\mathrm{OH}$ is formed in a highly excited state, and the excited $\mathrm{OH}$ rotational lines are produced by fluorescence. Similar processes may be at work in $\mathrm{T}$ Tauri disks, mediated by stellar FUV or X-ray radiation.

A last comparison between the present model calculations and the limited observations to test them involves $\mathrm{CO}$. According to Table 2, the model calculations predict comparable warm columns of $\mathrm{H}_{2} \mathrm{O}$ and $\mathrm{CO}$. The average ratio of water to $\mathrm{CO}$ to water columns in Table 2 is 1.6, independent of radius, with small variations of order 25\%. Carr \& Najita (2008) reported a ratio of water-to-CO columns of 1.3 for AA Tau, in rough accord with our model which ascribes most of the warm water and $\mathrm{CO}$ to the thermal-chemical transition region. The higher temperature and smaller emitting area of the $\mathrm{CO}$ lines compared to the water lines in AA Tau suggest that the $\mathrm{CO}$ and the water probe somewhat different radial distances. While the CO likely arises from small radii $(R<1 \mathrm{AU})$, the water may arise from radii out to $\gtrsim 2 \mathrm{AU}$ (Carr \& Najita 2008). The individual column densities of warm $\mathrm{CO}$ and water reported in Table 2 are consistent with the properties of the molecular emission reported for AA Tau.

It is also of interest to compare our results with other chemical calculations of the inner disk, e.g., Markwick et al. (2002), Agúndez et al. (2008), Woods \& Willacy (2009), and Gorti \& Hollenbach (2008). These comparisons are characterized by the fact that disk chemistry models differ widely in their underlying assumptions as well as in execution. None of these studies include mechanical heating, and only Woods and Willacy consider the recent theory of grain formation of $\mathrm{H}_{2}$ by Cazaux and Tielens (as do Nomura et al. 2009). For example, Markwick et al. (2002) reported only total molecular column densities with no information about the vertical abundance distribution. At $1 \mathrm{AU}$, they obtain $N\left(\mathrm{H}_{2} \mathrm{O}\right)=1.6 \times 10^{22} \mathrm{~cm}^{-2}$ and $N_{\mathrm{H}}=1.6 \times 10^{26} \mathrm{~cm}^{-2}$, for an average water abundance $x\left(\mathrm{H}_{2} \mathrm{O}\right)=10^{-4}$. Since they include only viscous heating, their disk atmosphere is much cooler than obtained in models that include stellar irradiation, and most of their calculated water column will not be warm. Markwick et al. do not discuss $\mathrm{OH}$.

Agúndez et al. (2008) developed a time-dependent model oriented toward the synthesis of complex molecules with neutral radical reactions. In Section 4 of their paper, they use the
D'Alessio (2001) model exposed to stellar and interstellar FUV radiation, but without $\mathrm{X}$-rays. They assume equal gas and dust temperatures and start with a molecular mixture with all oxygen in water that is not in CO. They obtain the following steady-state columns inside $3 \mathrm{AU}$, approximately independent of radius: $N(\mathrm{CO})=10^{18} \mathrm{~cm}^{-2}, N\left(\mathrm{H}_{2} \mathrm{O}\right) \approx 10^{17} \mathrm{~cm}^{-2}$ and $N(\mathrm{OH})=10^{14} \mathrm{~cm}^{-2}$. Although these values are similar to those calculated here, the molecules will not be as warm nor produce as much emission because the gas temperature is the same as the dust temperature.

Woods \& Willacy (2009) have developed a thermal-chemical model based on the D'Alessio (2001) disk structure that includes stellar and interstellar FUV radiation and X-rays. Their main emphasis is on the carbon isotopes, but they also report on the total water column density and give contours of water and $\mathrm{OH}$ in the inner disk. They show that the regions of the disk atmosphere where the average temperatures of the $\mathrm{OH}$ and water emission are the same as found for these species in AA Tau, have local abundances in the model that are similar to those reported by Carr \& Najita (2008). No quantitative information is provided on the column densities of warm $\mathrm{OH}$ and water.

Gorti \& Hollenbach (2008) have calculated a wide variety of atomic and molecular lines with a full thermal-chemical model that is similar to but more general than that used here. It includes X-rays as well as stellar FUV, although the $\mathrm{X}$-ray heating is significantly less than in our model. Gorti and Hollenbach also include cosmic rays, whereas we believe, by analogy with the much less powerful solar wind, that they are blown away from the inner protoplanetary disk by the stellar wind of the YSO (Glassgold et al. 1997). A key element of their model is its use of a significant population of small grains and polycyclic aromatic hydrocarbons (PAHs) to heat the gas via the photoelectric effect. However, they do not include mechanical heating, which we find plays an important role in the inner disk. Gorti and Hollenbach also simultaneously solve the equation for hydrostatic equilibrium, so that their disk atmosphere is more flared than ours. The column density of water is smaller than calculated here, and the line strengths are modest because the warm water is at smaller densities due to disk flaring (D. Hollenbach, private communication 2009). Consequently, they predict that only a few MIR lines should be detectable by the Spitzer IRS, in contrast with the rich emission spectra reported by Carr \& Najita (2008) and Salyk et al. (2008). On the other hand, we do not treat hydrostatic equilibrium here, and our future modeling will need to include it in order to assess its importance for the formation of $\mathrm{H}_{2}$ and the generation of significant columns of warm water.

\section{CONCLUSIONS}

Our results indicate that the conditions that lead to strong water emission in the infrared, i.e., large column densities of warm water molecules near the disk surface, can be produced in situ under certain conditions in an X-ray-irradiated disk model without requiring radial or vertical transport of water to the disk surface. In the inner disk $(\lesssim 0.25 \mathrm{AU})$, abundant warm water can be produced through gas-phase processes and only X-ray heating. Efficient $\mathrm{H}_{2}$ formation on warm grains and nonradiative heating appear to be capable of enlarging the radial range over which warm water emission is produced. These processes, when taken together, can account roughly for the column densities and emitting areas of the MIR water emission reported for T Tauri disks by Carr \& Najita (2008) and Salyk et al. (2008). 
We find that the column density (and temperature) of water at the disk surface depends significantly on the grain surface area in the disk atmosphere (i.e., on the extent of grain growth and settling) and on the heating of the atmosphere (and therefore perhaps on the disk accretion rate). Because both the disk accretion rate and the extent of grain settling are believed to vary among T Tauri disks, our model results suggest that the strength and character of MIR water emission from disks may also show some diversity. In contrast, because much less restrictive conditions are needed to produce large column densities of warm water close in $(<0.25 \mathrm{AU})$, water emission from this inner region of the disk may be quite common.

More specifically, for the grain areas that we use, we find that sources with modest amounts of grain growth (e.g., a factor of 20 decrease in area compared to the ISM) can produce large column densities of warm water within $1 \mathrm{AU}$. Even larger columns result for ten times more grain growth (see Table 2), and we might expect this trend to continue for a growth factor of 1000 . Recent studies of the MIR spectral energy distributions of T Tauri stars suggest that disks have experienced grain growth factors spanning a similar range (factors 10-1000; Furlan et al. 2006) as those considered in our models. Thus, we might expect that $\mathrm{T}$ Tauri disks that show different amounts of grain growth may display a diversity in the strength and character of their water emission.

Similarly, T Tauri disks are also believed to have accretion rates that vary by at least two orders of magnitude (Hartmann et al. 2006). Stellar X-ray luminosities also vary significantly. Since these factors influence the ionization and amount of surface heating, they may also contribute additional diversity to the water emission spectra of T Tauri disks. Large accretion rates can enhance the heating in the atmosphere and increase the strength of the water emission. A higher X-ray luminosity would produce both stronger ionization and heating. According to the simplified theory of Section 2.2, heating effects are more important than the ionization level. Such trends may be revealed in Spitzer IRS data sets that are being collected currently. However, if radial or vertical mixing is efficient, it might diminish or erase the correlations that would be predicted by our models.

In addition to searching for correlations in large data sets, it may also be interesting to explore individual objects in greater detail in the context of our models. In the case of AA Tau, the analysis of Furlan et al. (2006) suggests that the dust in the disk surface may be depleted by a factor of nearly 1000 . Our calculations suggest that the observed warm water columns can be accounted for, even for this degree of depletion, without nonradiative heating. Thus, in this case, mixing and transport appear to be unnecessary to account for the observations.

We have identified two major uncertainties in the chemistry of $\mathrm{H}_{2}$ that bear on the amount of water and other species.

1. Three-body formation of $\mathrm{H}_{2}$. Although the use by Flower and Harris of a new equilibrium constant represents an important step forward, the data on which these reactions are based are old measurements of the low-energy collisional excitation of $\mathrm{H}_{2}$ by atomic hydrogen (Jacobs et al. 1967; Baulch et al. 2005). New experiments are needed.

2. Grain formation of $\mathrm{H}_{2}$. The adopted rate coefficients are based on theory (Cazaux \& Tielens 2002, 2004). Although this model is consistent with observations of $\mathrm{H}_{2}$ lines in PDRs (Habart et al. 2004; Cazaux \& Tielens 2006), its applicability to the dense and dust-depleted atmospheres of protoplanetary disks is not yet fully established.
Regarding nonradiative heating, we have used Equation (18) from GNI04, who suggested two ways of heating the transition region above the level achievable with X-rays, dissipation of turbulence and the interaction of the stellar wind with the disk atmosphere. A sound basis for these processes has not yet been laid, but progress should be possible with improved numerical simulations of the magnetorotational instability (e.g., Turner \& Sano 2008; Terquem 2008) and of the wind-disk interaction (e.g., Li \& Shu 1996; Matsuyama et al. 2009). Our results highlight the need for further basic studies of the heating generated by the MRI and by the interaction of the wind and the disk.

Given these current uncertainties, and the potential sensitivity of water emission to multiple effects (X-ray irradiation, grain growth, disk accretion, transport, etc.), it may be difficult to diagnose the role of such important (but elusive) processes as radial and/or vertical transport from observations of water alone. It would be worthwhile to establish diagnostics for other relevant regions of a protoplanetary disk such as the disk midplane and the outer disk. An interesting example is provided by the discussion by Woods \& Willacy (2007) of the formation of benzene near the midplane of the inner part of a protoplanetary disk.

This work has been supported by NSF grant AST-0507423, NASA grants NNG06GF88G and 1322305 to UC Berkeley. We would like to thank John Black, David Hollenbach, and Xander Tielens for discussions of chemical processes in disks and David and Xander for helpful comments on our manuscript.

\section{APPENDIX}

\section{PAH FORMATION OF $\mathrm{H}_{2}$ IN PROTOPLANETARY DISKS}

Duley \& Williams (1993) suggested that $\mathrm{H}_{2}$ forms on PAHs in carbonaceous interstellar dust, and Bauschlicher (1998) demonstrated that it was energetically favorable for PAH cations to acquire an $\mathrm{H}$ atom and then form $\mathrm{H}_{2}$ in a subsequent collision with atomic $\mathrm{H}$. He suggested that this was a way to synthesize $\mathrm{H}_{2}$ in the gas phase. Rauls \& Hornekær (2008) have recently discussed how superhydrogenated neutral PAHs might also play a role in the gas-phase synthesis of $\mathrm{H}_{2}$. Jonkheid et al. (2006) included $\mathrm{H}_{2}$ formation by PAHs in modeling the transitional disk around HD 141569. Habart et al. (2004) adduced possible evidence for $\mathrm{H}_{2}$ formation by PAHs in PDRs.

As a basis for evaluating the possibility that PAH formation of $\mathrm{H}_{2}$ is relevant in the molecular transition region of disks, we make a crude estimate of the rate coefficient for the Bauschlicher process,

$$
\left(\mathrm{PAH}^{+}+\mathrm{H}\right)+\mathrm{H} \rightarrow \mathrm{PAH}^{+}+\mathrm{H}_{2},
$$

where the symbol $\left(\mathrm{PAH}^{+}+\mathrm{H}\right)$ represents a stable $\mathrm{PAH}$ cation with an added $\mathrm{H}$ atom. The effective rate coefficient $R_{\mathrm{PAH}}$ for this process, which can then be compared with the rate coefficient $R$ for grain formation in Equation (15), can be written as,

$$
R_{\mathrm{PAH}}=k_{\mathrm{PAH}} f\left(\mathrm{PAH}^{+}+\mathrm{H}\right) x_{\mathrm{PAH}},
$$

where $k_{\mathrm{PAH}}$ is the unknown rate coefficient for reaction $\mathrm{A} 1$, $f\left(\mathrm{PAH}^{+}+\mathrm{H}\right)$ is the fraction of appropriately hydrogenated PAH cations, and $x_{\mathrm{PAH}}$ is the total gas-phase abundance of PAHs in all ionization states. We make a rough guess that $k_{\mathrm{PAH}} \sim 10^{-10} \mathrm{~cm}^{3} \mathrm{~s}^{-1}$ and assume that $f\left(\mathrm{PAH}^{+}+\mathrm{H}\right) \sim 10^{-2}$ and $x_{\mathrm{PAH}} \sim 10^{-7}$, an upper limit to the range deduced by Geers 
et al. (2006) from Spitzer observations of disks. The result, $R_{\text {PAH }} \sim 10^{-19} \mathrm{~cm}^{3} \mathrm{~s}^{-1}$, is only slightly smaller than given by Equation (15) for grain formation of $\mathrm{H}_{2}$.

Each of the factors in our estimate of $R_{\mathrm{PAH}}$ is uncertain by at least 1 dex, so we cannot rule $\mathrm{PAH}$ formation of $\mathrm{H}_{2}$ in or out on this basis. However, we suspect that it is unimportant for the inner regions of protoplanetary disks because PAHs close to YSOs are likely to be destroyed by stellar X-rays. We estimated the total abundance $x_{\mathrm{PAH}} \sim 10^{-7}$ on the basis of the low detection frequency of PAH IR features in Spitzer observations of T Tauri stars (Geers et al. 2006; 10\%) and Class I YSOs (Geers et al. 2009; $\lesssim 2 \%$ ). Even where observed, the PAHs may occur mainly at large radii, as for Herbig stars (Habart et al. 2006; Geers et al. 2007; Goto et al. 2008). Laboratory experiments with the Synchrotron Radiation Facility in Grenoble show that PAHs and very small particles are destroyed by hard X-rays (Gougeon 1998; Mitchell et al. 2002; see also Section 6 of the review by Glassgold et al. 2000). Indeed, Voit (1992) invoked $\mathrm{X}$-ray destruction of PAHs to explain the difference between the observed PAH features seen in starburst galaxies and AGN. Finally, Siebenmorgen \& Kreugel (2008) have recently modeled the PAH abundance in T Tauri disks and found that energetic photons destroy PAHs inside of 10 AU. It is for these reasons in support of a low PAH abundance that we tentatively ignore $\mathrm{PAH}$ formation of $\mathrm{H}_{2}$ for the inner part of the atmospheres of protoplanetary disks.

\section{REFERENCES}

Agúndez, M., Cernichara, J., \& Goicoechea, 2008, A\&A, 483, 831

Andersson, S., Al-Halabi, A., Kroes, G.-J., \& van Dishoeck, D. F. 2008 , J. Chem. Phys., 124, 064715

Anicich, V. 1993, J. Phys. Chem. Ref. Data, 22, 1469

Baulch, et al. 1992, J. Phys. Chem. Ref. Data, 21, 411

Baulch, et al. 2005, J. Phys. Chem. Ref. Data, 34, 757

Bauschlicher, C. W. 1998, ApJ, 509, L125

Bergin, E., Calvet, N., D’Alessio, P., \& Herczeg, G. J. 2003, ApJ, 591, L159

Burke, J. R., \& Hollenbach, D. J. 1983, ApJ, 265, 223

Carr, J. S., \& Najita, J. 2008, Science, 319, 2008

Carr, J. S., Tokunaga, A., \& Najita, J. 2004, ApJ, 603, 213

Cazaux, S., Caselli, P., Tielens, A. G. G. M., Le Bourlet, J., \& Walmsley, M. 2005, J. Phys. Conf. Ser., 6, 155

Cazaux, S., Caselli, P., Walmsley, M., \& Tielens, A. G. G. M. 2006, in Astrochemistry: Recent Successes and Current Challenges, ed. D. C. Lis, G. A. Blake, \& E. Herbst (Cambridge: Cambridge Univ. Press), 325

Cazaux, S., \& Tielens, A. G. G. M. 2002, ApJ, 575, L29

Cazaux, S., \& Tielens, A. G. G. M. 2004, ApJ, 604, 222

Ciesla, F. J., \& Cuzzi, J. N. 2006, Icarus, 181, 178

Cohen, N., \& Westberg, K. R. 1983, J. Phys. Chem. Ref. Data, 12, 531

D’Alessio, P., Calvet, N., \& Hartmann, L. 2001, ApJ, 553, 321

D’Alessio, P., Calvet, N., Hartmann, L., Lizano, S., \& Cantó, J. 1999, ApJ, 527, 893

Duley, W. W., \& Williams, D. A. 1993, MNRAS, 260, 37

Faure, A., \& Josselin, E. 2008, A\&A, 492, 25

Fields, D., Adams, N. G., \& Smith, D. 1980, MNRAS, 192, 1

Flower, D. R., \& Harris, G. J. 2007, MNRAS, 377, 705

Furlan, E., et al. 2006, ApJS, 165, 568

Geers, V. C., van Dishoeck, E. F., Pontoppidan, K. M., Lahuis, F., Crapsi, A., Dullemond, C. P., \& Blake, G. A. 2009, A\&A, 495, 837

Geers, V. C., van Dishoeck, E. F., Visser, R., Pontoppidan, K. M., Augereau, J.-C., Habart, E., \& Lagrange, A. M. 2007, A\&A, 476, 279

Geers, V. C., et al. 2006, A\&A, 459, 545

Glassgold, A. E., Feigelson, E. D., \& Montmerle, T. 2000, in Protostars \& Planets IV, ed. V. Mannings, A. P. Boss, \& S. Russell (Tucson, AZ: Univ. Arizona Press), 429
Glassgold, A. E., Najita, J., \& Igea, J. 1997, ApJ, 480, 344

Glassgold, A. E., Najita, J., \& Igea, J. 2004, ApJ, 615, 972 (GNI04)

Gorti, U., \& Hollenbach, D. 2004, ApJ, 613, 424

Gorti, U., \& Hollenbach, D. 2008, ApJ, 683, 287

Goto, M., et al. 2008, ApJ, 693, 610

Gougeon, S. 1998, Thèse de doctorat, Univ. of Paris VII

Grosso, N., Montmerle, T., Fernández, Granklin, K., \& Zapatero, O. M. R. 2007, A\&A, 475, 607

Habart, E., Boulanger, F., Verstraete, L., Walmsey, C. M., \& Pineau des Forêts, G. 2004, A\&A, 414, 531

Habart, E., Natta, A., Testi, L., \& Carbillet 2006, A\&A, 449, 1067

Hartmann, L., D’Alessio, P., Calvet, N., \& Muzerolle, J. 2006, ApJ, 648, 484

Hollenbach, D. J., \& Salpeter, E. E. 1971, ApJ, 163, 155

Hornekær, L., et al. 2006a, Phys. Rev. Lett., 96, 156104

Hornekær, L., et al. 2006b, Phys. Rev. Lett., 97, 186102

Jacobs, T. A., Giedt, R. R., \& Cohen, N. 1967, J. Chem. Phys., 47, 54

Jewitt, D., Chizmadia, L., Grimm, R., \& Prialnik, D. 2006, in Protostars and Planets V, ed. B. Reipurth, D. Jewitt, \& K. Keil (Tucson, AZ: Univ. Arizona Press), 863

Jonkheid, B., Kamp, I., Augereau, J.-C., \& van Dishoeck, E. F. 2006, A\&A, 453,163

Knez, C., et al. 2007, BAAS, 39, 812

Krolik, J. H., \& Kallman, T. R. 1983, ApJ, 267, 610

Lepp, S., \& Dalgarno, A. 1996, A\&A, 306, L21

Li, Z.-Y., \& Shu, F. H. 1996, ApJ, 468, 261

Maloney, P. R., Hollenbach, D. J., \& Tielens, A. G. G. M. 1996, ApJ, 4446, 561

Mandell, A., Mumma, M. J., Blake, G. A., Bonev, B. P., Villaneuva, G. L., \& Salyk, C. 2008, ApJ, 681, L25

Markwick, A. J., Ilgner, M., Millat, T. J., \& Henning, Th. 2002, A\&A, 385, 632

Matsuyama, I., Johnstone, D., \& Hollenbach, D. 2009, arXiv:0904.3361v1

Meijerink, R., Glassgold, A. E., \& Najita, J. R. 2008, ApJ, 676, 518

Meijerink, R., \& Spaans, M. 2005, A\&A, 436, 397

Mitchell, J. B. A., Rebrion-Rowe, C., LeGarrec, J.-L., Taupier, G., Huby, N., \& Wulff, M. 2002, A\&A, 86, 743

Najita, J. R., Carr, J. S., Glassgold, \& Valenti, J. A. 2007, in Protostars and Planets V, ed. B. Reipurth, D. Jewitt, \& K. Keil (Tucson, AZ: Univ. Arizona Press), 507

Nomura, H., Aikawa, Y., Nakagawa, Y., \& Millar, T. J. 2009, A\&A, 495, 183

Nomura, H., Aikawa, Y., Tsujimoto, M., Nakagawa, Y., \& Millar, T. J. 2007, ApJ, 661,334

Nomura, H., \& Millar, T. J. 2005, A\&A, 438, 923

Perets, H. B., Biham, O., Manicó, G., Pirronello, V., Roser, J., Swords, S., \& Vidali, G. 2005, ApJ, 627, 850

Perets, H. B., et al. 2007, ApJ, 661, L163

Rauls, E., \& Hornekær, R. L. 2008, ApJ, 679, 531

Salyk, C., Pontippidan, K. M., Blake, G. A., Lajuis, F., van Dishoeck, E. F., \& Evans, N. J. 2008, ApJ, 676, L49

Siebenmorgen, R., \& Kreugel, E. 2008, Heidelberg Symp. on Radiative Transfer, www.mpia.de/RT08/par.htm

Stauber, P., Doty, S. D., van Dishoeck, E. F., \& Benz, A. O. 2005, A\&A, 440, 949

Stauber, P., Jorgensen, J. J., an Dishoeck, E. F., Doty, S. D., \& Benz, A. O. 2006, A\&A, 453, 555

Tappe, A., Lada, C. J., Black, J. H., \& Muench, A. A. 2008, ApJ, 680, L117

Terquem, C. E. J. M. L. J. 2008, ApJ, 689, 532

Turner, N. J., \& Sano, T. 2008, ApJ, 679, L131

van Dishoeck, E. F., \& Dalgarno, A. 1983, J. Chem. Phys., 79, 873

van Dishoeck, E. F. 2004, ARA\&A, 42, 119

Vidali, G., Roser, J. E., Manicó, G., \& Pirronello, V. 2004, J. Geophys. Res., 109, E07S14

Voit, G. M. 1992, MNRAS, 258, 841

Wood, K., Smith, D., Whitney, B., Stassun, K., Kenyon, S. J., Wolff, M. J., \& Bjorkman, K. S. 2001, ApJ, 561, 299

Woodall, J., Agúndez, M., Markwick-Kemper, A. J., \& Millar, T. J. 2007, A\&A, 466, 1197

Woods, P. M., \& Willacy, K. 2007, ApJ, 655, L49

Woods, P. M., \& Willacy, K. 2009, ApJ, 693, 1360

Zecho, T., et al. 2002, Chem. Phys. Letts., 366, 188 